\title{
A Systematic Review of the Technology Acceptance Model in Health Informatics
}

\author{
${ }^{1}$ Department of Health Information Technology, School of Allied \\ Medical Sciences, Urmia University of Medical Sciences, Urmia, Iran \\ 2 Student Research Committee, Urmia University of Medical Sciences, \\ Urmia, Iran \\ 3 Department of Computer and Information Sciences, Linköping \\ University, Linköping, Sweden \\ ${ }^{4}$ Department of Medical and Health Sciences, Linköping University, \\ Linköping, Sweden
}

Bahlol Rahimi ${ }^{1}$ Hamed Nadri ${ }^{1,2}$ Hadi Lotfnezhad Afshar ${ }^{1}$ Toomas Timpka ${ }^{3,4}$

Appl Clin Inform 2018;9:604-634.
Address for correspondence Hamed Nadri, MSc, Department of Health Information Technology, School of Allied Medical Sciences, Urmia University of Medical Sciences, Nazloo Campus, Sero Road, Urmia, Iran (e-mail: Hamednadri4@gmail.com).

\section{Abstract}

Keywords

- technology acceptance model

- literature review

- health information technology

- technology acceptance

- theoretical models

- health informatics
Background One common model utilized to understand clinical staff and patients' technology adoption is the technology acceptance model (TAM).

Objective This article reviews published research on TAM use in health information systems development and implementation with regard to application areas and model extensions after its initial introduction.

Method An electronic literature search supplemented by citation searching was conducted on February 2017 of the Web of Science, PubMed, and Scopus databases, yielding a total of 492 references. Upon eliminating duplicates and applying inclusion and exclusion criteria, 134 articles were retained. These articles were appraised and divided into three categories according to research topic: studies using the original TAM, studies using an extended TAM, and acceptance model comparisons including the TAM.

Results The review identified three main information and communication technology (ICT) application areas for the TAM in health services: telemedicine, electronic health records, and mobile applications. The original TAM was found to have been extended to fit dynamic health service environments by integration of components from theoretical frameworks such as the theory of planned behavior and unified theory of acceptance and use of technology, as well as by adding variables in specific contextual settings. These variables frequently reflected the concepts subjective norm and self-efficacy, but also compatibility, experience, training, anxiety, habit, and facilitators were considered.

Conclusion Telemedicine applications were between 1999 and 2017, the ICT application area most frequently studied using the TAM, implying that acceptance of this technology was a major challenge when exploiting ICT to develop health service organizations during this period. A majority of the reviewed articles reported extensions of the original TAM, suggesting that no optimal TAM version for use in health services has been established. Although the review results indicate a continuous progress, there are still areas that can be expanded and improved to increase the predictive performance of the TAM. received

February 5, 2018

accepted after revision

June 24, 2018 (c) 2018 Georg Thieme Verlag KG Stuttgart · New York
DOI https://doi.org/

10.1055/s-0038-1668091.

ISSN 1869-0327. 


\section{Background and Significance}

New technologies are continuously being adopted in health services. ${ }^{1,2}$ Modern information and communication technology (ICT) has been understood to improve service quality in the health service sector in general and in clinical medicine and at hospitals in particular, enhancing patient safety, staff efficiency and effectiveness, and reducing organizational expenses. ${ }^{3-6}$ Meanwhile, progress in the life sciences has led to higher medical specialization and needs to exchange health information across institutional borders. ${ }^{7,8}$ Despite these needs, health information systems development methods and research have focused on the technical aspects of the system design. ${ }^{9-13}$ If the latter efforts are insufficient to meet the needs of progressive health service organizations and individual users, ICT investments will be spent ineffectively, and, potentially, patients put at risk. ${ }^{14}$ Therefore, the impact on ICT adoption of different nontechnical and individual-level factors need to be established. ${ }^{15}$ In this regard, it is positive that technology acceptance studies at the present are considered to stand as a mature field in information systems research. ${ }^{16}$

During the past 30 years, several theoretical models have been proposed to assess and explain acceptance and behaviors in association with ICT introduction. Robust measures have been developed of how well a technology "fits" with user tasks and have validated these task-technology fit instruments. ${ }^{17}$ The best known of these is the technology acceptance model (TAM), which was presented in $1989,{ }^{18}$ and has during this period been applied and empirically tested in a wide spectrum of ICT application areas. ${ }^{19,20}$ Also, the TAM is one of the most popular research models to predict use, person's intention to perform a particular behavior, and acceptance of information systems and technology by individual users. ${ }^{21,22}$ Originally, the TAM was derived from the social psychological theories of reasonable action (TRA) and planned behavior (TPB), ${ }^{23}$ these three models focus on a person's intention to perform the behavior, ${ }^{24}$ but the constructs of these three models are different and not exactly the same. The TAM has become the dominant model for investigating factors affecting users' acceptance of novel technical systems. ${ }^{25}$ The basic model presumes a mediating role of perceived ease of use and usefulness in association between system characteristics (external variables) and system usage (as shown in -Fig. 1). ${ }^{26}$ Several reviews of TAM use encompassing the ICT field in total have been issued. Accounts of the first decade of TAM-related research and suggestions of future directions were offered in 2003 by Lee et $\mathrm{al}^{27}$ and Legris et al. ${ }^{25}$ The directions included a need for incorporating more variables related to human and social change processes and exploring boundary conditions. At that time, the original TAM had already been modified in the TAM2 version ${ }^{28}$ by removal of the "Attitudes" concept and differentiating the "External variables" concept into social influence (subjective norm, voluntariness, and image), cognitive instrumental processes (job relevance, output quality, and result demonstrability), and experience. A few years later, Sharp continued to discuss the relative strengths of perceived usefulness (PU) and perceived ease and the role of attitudes in user acceptance, but also brought to the fore differences between volitional and mandatory use environments. ${ }^{29}$ Venkatesh et al proposed a unified modelthe unified theory of acceptance and use of technology (UTAUT)-based on studies of eight prominent models (in particular the TAM). The UTAUT is formulated with four core determinants of intentions and usage: performance expectancy, effort expectancy, social influence, and facilitating conditions, together with four moderators of key relationships: gender, age, experience, and voluntariness of use. ${ }^{16}$ The same year, King and Jun conducted a statistical metaanalysis of TAM applications in various fields, reporting the TAM to be a valid and robust model that has been widely used. $^{30}$ In 2008, the TAM2 was extended with regard to determinants of perceived ease of use (PEOU) (TAM3). ${ }^{31}$ The TAM3 is composed of four constructs: PEOU, PU, behavior intention, and use behavior.

Turning the attention from theory building to use environments, Turner et al concluded that care should be taken when using a particular version of the TAM outside the context in which the version originally was validated. ${ }^{32}$ Proceeding with the analyses of model validity across use environments, Hsiao and Yang used cocitation analyses to identify three main application contexts for TAM use: (1) task-related systems, (2) e-commerce systems, and (3) "experiential" (or "hedonic") systems. ${ }^{33}$

Task-related systems are designed to improve task performance and efficiency. These systems can be categorized as automation software, office systems, software development, and communication systems such as electronic health record (EHR). Clinical practice guidelines, linked educational content, and patient handouts can be part of the EHR. This may permit finding the answer to a medical question while the

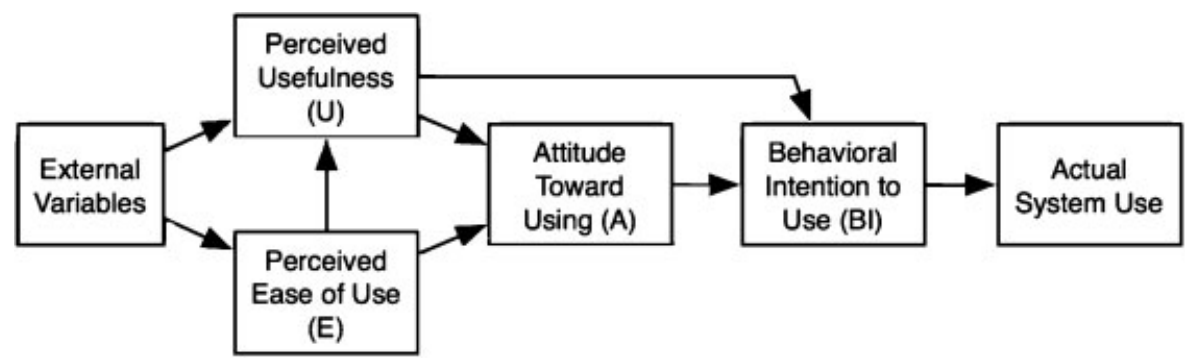

Fig. 1 The basic technology acceptance model. ${ }^{18}$ 
patient is still in the examination room. ${ }^{34} \mathrm{e}$-Commerce is the activity of buying or selling of products on online services or over the Internet. ${ }^{35}$ The "hedonic" information systems are usually connected to home and leisure activities, focusing on the fun or novel aspect of information systems includes online gaming, online surfing, online shopping, and even online learning while perusing enjoyment at the same time. ${ }^{33}$

In 2010, Gagnon et al conducted a systematic review to investigate factors influencing the adoption of ICT by health care professionals. In this review, including all ICT acceptance models in health services, it was concluded that PU of system and PEOU were the two most influential factors. ${ }^{36}$ These two factors are the main components of the original TAM. $^{22}$ Regarding applications in specific health services areas, Strudwick concluded from a review of TAM applications among nursing practitioners that a modified TAM with variables detailing the health service context and user groups added could provide a better explanation of nurses' acceptance of health care technology. ${ }^{37}$ Further, Ahlan and Isma'eel reported from an overview of patient acceptance of ICT that the TAM is one of the most useful models for studying patients' perceptions and behaviors. ${ }^{38}$ Also, Garavand et al concluded from their general review of the most widely used acceptance models in health services that the TAM is the most important model used to identify the factors influencing the adoption of information technologies in the health system. ${ }^{39}$

\section{Objective}

The objective of this systematic review was to compile published research on TAM use in health information systems development and implementation with regard to application areas and model modifications after its initial introduction, and also to gain understanding of the existing research and debates relevant to a particular topic or area of study. In the present setting, the development of health services requires parallel adjustments of ICT support, and accordingly, of TAMs.

\section{Method}

We used systematic search processes to identify all published original articles related to TAM applications in health services from 1989, the year when the TAM was introduced, to February 2017. The PubMed, Scopus, and Web of Science databases were searched and English-only publications selected. The broad keywords used for the initial search are displayed in -Table 1. The authors, title, journal, year of publication, and abstract for each article were collected in an Excel spreadsheet. First, the publication's titles, and abstracts, were assessed together by two of the four authors, after reviewing all abstracts and eliminating those categorized with exclusion criteria or lacking inclusion criteria; the full texts of the relevant articles were then reviewed by three authors together. The full texts of the remaining articles were read for eligibility, and the qualified publications were
Table 1 Terms used in search

\begin{tabular}{|l|l|l|}
\hline Keyword & Boolean & Additional keywords \\
\hline $\begin{array}{l}\text { Technology } \\
\text { acceptance model } \\
\text { (TAM) }\end{array}$ & AND & Healthcare \\
\hline $\begin{array}{l}\text { Technology } \\
\text { acceptance model, } \\
\text { TAM, hospital } \\
\text { information system } \\
\text { (HIS), extended } \\
\text { technology } \\
\text { acceptance model, } \\
\text { TAM2, TAM3 }\end{array}$ & AND & $\begin{array}{l}\text { Healthcare, medicine, } \\
\text { health information } \\
\text { system (HIS), } \\
\text { telemedicine, } \\
\text { telehealth, electronic } \\
\text { health record (EHR), } \\
\text { computerized } \\
\text { physician medication } \\
\text { order entry (CPOE), } \\
\text { medication system, } \\
\text { bar code medication } \\
\text { administration } \\
\text { (BCMA) }\end{array}$ \\
\hline
\end{tabular}

retained in a list. A search of the recent reviews and handsearching references from articles were made to get related articles. The TAM has been used in many technological and geographical contexts. Several major technologies like mobile and telemedicine have variety of applications. ${ }^{40,41}$ In a separate phase, the technologies and applications as a subset of major technological contexts and characteristics of each tested model for user groups were identified by three authors together. Finally, the publications in the list were classified into three categories according to their aim and content:

- Original TAM: Applications of the original TAM. In this category, the relationship between the main constructs of the original TAM is examined. These relationships include the relationship between $\mathrm{PU}$ and perceived ease to use with intention to use and also the relationship between perceived ease to use and PU.

- Development and Extension of TAM: Reports of new insights related to the core elements of TAM and/or development of new TAM versions by integrating new factors and other acceptance theory variables with the original TAM. These factors incorporate into the constructs of the original TAM as predictive and moderating variables.

- Comparisons of the TAM with other technology acceptance models: The TAM and other theoretical models are compared by examining factors associated with the adoption of a particular technology.

\section{Results}

A total of 492 document references were retrieved from the database searches. After removal of 44 duplicates, 448 publications were entered into the selection process. Results of the screening process in the analysis are noted in the flow diagram in -Fig. 2. First, 448 publications' titles and abstracts were assessed together by two of the four authors. At this stage, 120 articles unrelated to the topic were excluded from the review. The full texts of the relevant 


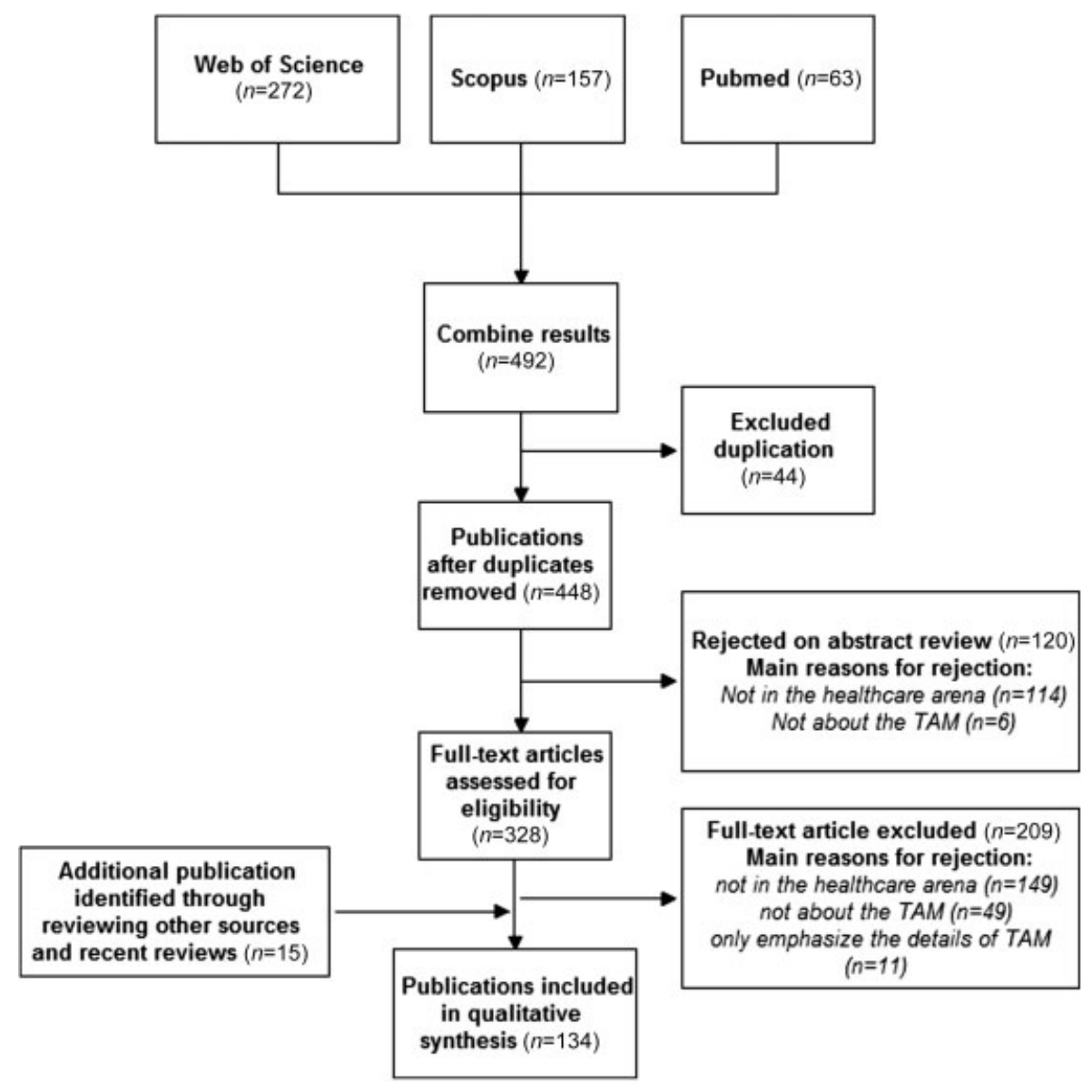

Fig. 2 Flow diagram of the study.

articles were then reviewed by three authors together. The titles and abstracts of the relevant articles were then reviewed by three authors. When the title or abstract was deemed significant for inclusion in the review, the full text was scanned to ensure that the content was relevant. At this stage, 209 articles that were unrelated to acceptance of technology in health care, TAM constructs, or only addressed separate components of the TAM and other acceptance models were excluded. When there was disagreement, the authors evaluated their assessment until consensus was reached. A search of the recent reviews and hand-searching references from articles yielded an additional 15 papers. The systematic search of the literature identified 134 articles that reported original empirical research on the use of the TAM within health services.

Publications dealing with the original TAM had peaked ( $n=3,2.2 \%$ of all articles) in 2013 and 2015, publications on development and extension of TAM peaked ( $n=16,11.9 \%)$ in 2013, while publications reporting comparisons of TAMs had peaked ( $n=2,1.5 \%$ ) in years 2010 and 2013 (-Fig. 3). A general increase in reports of TAM use suggests a persisting interest in understanding technology acceptance in health services. Also, there was a noteworthy leap in reports of TAM extensions in 2012 (-Fig. 3), which implies a recent highlighting of the influence from external factors on technology acceptance. The 134 articles reporting on TAM use had been published in 72 scientific journals, and originated from 30 countries; 29 (21.6\%) studies from the United States, 28 (20.9\%) from Taiwan, 14 (10.4\%) from Spain, while the remaining articles originated from countries in Europe, Asia, and Africa. The journals with the highest numbers of articles were International Journal of Medical Informatics with 11 studies (8.14\%), Telemedicine and e-Health with 10 studies (7.4\%), and BMC Medical Informatics and Decision Making, with 8 studies (5.9\%).

The first study of a TAM use in health services was reported in $1999,{ }^{42}$ analyzing physicians' intentions associated with the adoption of the telemedicine technology in a Hong Kong hospital setting. The ICT application area in which the TAM was first more frequently applied was EHR for which a peak in publications was observed in 2009. Publications reporting the TAM applications in telemedicine reached its peak in 2014, while the use of the TAM for analyses of mobile applications did peak in 2015. The first integration of several acceptance models with the TAM in health services was reported from Finland for examining acceptance of mobile systems among physicians. ${ }^{43}$ In this study, the TAM was combined with the UTAUT and Personal Innovativeness in the Domain of Information Technology (PIT) models.

Three main technological contexts were identified for applications of the TAM (-Table 2): (1) Telemedicine with 25 studies (18.6\%), (2) EHR with 21 studies (15.7\%), and (3) 


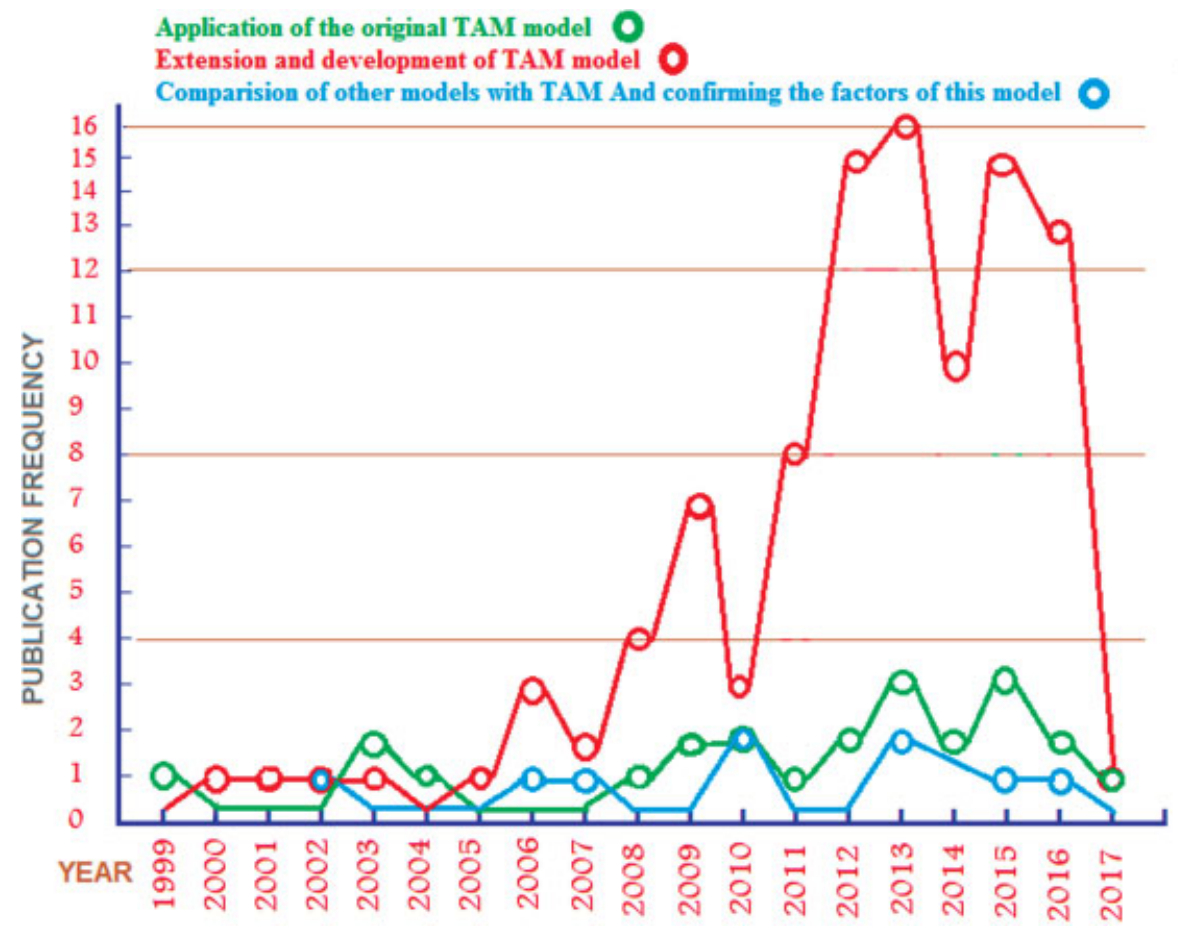

Fig. 3 Frequency of articles reporting technology acceptance model use according to the three study categories displayed by year.

mobile applications with 15 studies (11.2\%). Researchers in different countries have focused on different specific technologies: researchers in Taiwan on telemedicine (8 articles), mobile applications $(n=5)$, and hospital information systems (HIS) $(n=4)$; in the United States on EHRs $(n=8)$, computers, handheld (personal digital assistants [PDAs]) $(n=4)$, telemedicine, and personal health records $(n=2)$; and in Spain on telemedicine $(n=6)$, while researchers from Iran have focused on $\operatorname{EHR}(n=3)$ technology (-Fig. 4).

Telemedicine, the area where the TAM has been most widely applied, is also the first technology that was studied using the TAM (-Fig. 5). TAM application on mobile technologies was initiated in $2006^{43}$ and these studies peaked in 2015. As shown in - Table 3, most studies have emphasized the acceptance of physicians $(n=43,32 \%)$ and nurses ( $n=34,25.3 \%)$. Other users of technology acceptance include patients and clients of health services, pharmacists, and other medical professionals.

\section{Applications of the Original TAM}

As shown in -Table 4, 23 (17.1\%) of the identified articles reported application of the original TAM. In most studies using the original TAM to assess technology acceptance, the main constructs (i.e., PU and perceived ease to use) of TAM were supported. The most frequent ICT application areas were telemedicine, $n=6$ (26\%) and PDA, $n=2$ (8.6\%). The study participants ranged from 10 to 1,942 , with an average of 184 . The user category involved in the most studies was nurses $(n=4,17 \%)$ followed by physicians and patients (both $n=3,13 \%$ ).

\section{Development and Extension}

Of all studies, $102(76.1 \%)$ studies reported development or extension of the TAM. In these studies, different factors and theories were incorporated to the original TAM ( - Table 5). The factors investigated in the most commonly used technological contexts such as health information technology systems in general, telemedicine, EHR, mobile apps, HIS, Eprescription, PDAs, and personal health record are briefly provided. According to the results in various technological contexts, it is possible to draw basic factors that incorporate with the original TAM for each technological context. The most common factors added to the TAM in almost all technological contexts were, in order of importance and frequency of repetition, compatibility, subjective norm, self-efficacy, experience, training, anxiety, habit, and facilitators. These factors can be a basic model for most technological contexts with the incorporation of the original TAM and separate variables regarding a context.

Adding separate variables to develop contextualized TAM versions allows optimizing specific dimensions of the TAM in particular settings and thereby improving predictions in these contexts. A full summary of the additions to the original TAM displayed by technology application area in health services, theories integrated, and new factors and variables inserted is shown in - Table 6 . The most commonly integrated theories were classic acceptance models such as UTAUT, TRA, Diffusion of Innovation theory, and the TPB. In addition to the theories, the conditions and technologies forming the particular context in specific settings have been used to add further concepts and variables, i.e., some factors were not derived from any technology acceptance theory and were instead specific to a certain technology (such as 
Table 2 Numbers of articles analyzing ICT adoption using TAM (main topics according to the MeSH thesaurus)

\begin{tabular}{|l|l|l|}
\hline Main topic (MeSH) & Number & $\begin{array}{l}\text { Directions of } \\
\text { country based on } \\
\text { technology }\end{array}$ \\
\hline Telehealth & 25 & $\begin{array}{l}\text { Taiwan (8), Spain (6), } \\
\text { United States (2) }\end{array}$ \\
\hline Electronic health record & 21 & $\begin{array}{l}\text { United States (8), } \\
\text { Iran (3) }\end{array}$ \\
\hline Mobile applications & 15 & Taiwan (5) \\
\hline HIT systems in general & 8 & - \\
\hline Computers, handheld & 7 & United States (4) \\
\hline $\begin{array}{l}\text { Hospital information } \\
\text { systems }\end{array}$ & 6 & Taiwan (4) \\
\hline $\begin{array}{l}\text { Decision support } \\
\text { systems, clinical }\end{array}$ & 5 & - \\
\hline Electronic prescribing & 4 & - \\
\hline Health records, personal & 4 & United States (2) \\
\hline $\begin{array}{l}\text { Automatic data } \\
\text { processing (bar code) }\end{array}$ & 3 & - \\
\hline $\begin{array}{l}\text { Radiology information } \\
\text { systems }\end{array}$ & 2 & - \\
\hline $\begin{array}{l}\text { Medical order entry } \\
\text { systems }\end{array}$ & 2 & - \\
\hline $\begin{array}{l}\text { Management information } \\
\text { systems }\end{array}$ & 2 & - \\
\hline $\begin{array}{l}\text { Clinical information } \\
\text { system }\end{array}$ & 2 & - \\
\hline $\begin{array}{l}\text { Enterprise resources } \\
\text { planning }\end{array}$ & 2 & - \\
\hline The remaining of the studies dealt with one technology each \\
\hline
\end{tabular}

Abbreviations: CPOE, computerized physician order entry; HIT, health information technology; ICT, information and communication technology; MeSH, Medical Subject Headings; PACS, picture archiving and communication system; PDA, personal digital assistant; TAM, technology acceptance model.

Note: The parenthesized value is number of studies.

technology features, environmental conditions, user types, etc.). Among the 102 articles, only two studies were conducted on the TAM3.

\section{Comparison of Other Technology Acceptance Models with TAM}

Nine (6.7\%) studies compared TAM with other TAMs. The most common ICT application area for these comparisons was mobile technology, $n=3$ (33.3\%). Typically, Hsiao and Tang $^{44}$ used different variables to investigate the introduction of mobile technologies from the perspective of the elderly people in Taiwan. Their results supported the validity of the TAM variables, and also the inclusion of novel factors such as perceived ubiquity, personal health knowledge, and perceived need for health care. Day et $\mathrm{al}^{45}$ conducted a study to evaluate hospice providers' attitudes and perceptions regarding videophone technology in settings where the technology was introduced but underutilized. Findings indicate that the TAM provides a good framework for an understanding of telehealth underutilization.

In two studies on telemedicine acceptance among physicians in China and the United States, respectively, the TAM and the TPB model were compared. Interestingly, the findings from China suggested that the TAM was more valid than the TPB, while the TPB was more valid than the TAM in the United States. ${ }^{46,47}$ Another study comparing the TAM and the UTAUT among physicians concluded that the usage intentions were strongly associated with the performance expectancy on attitude and attitude concepts. ${ }^{48}$ Manimaran and Lakshmi $^{49}$ formulated an integrated TAM for Health Management Information System and concluded that health workers' innovativeness and voluntariness had a direct and positive influence on these intentions. Similarly, Smith and Motley $^{50}$ found that e-prescribing acceptance was predicted by the technological sophistication, operational factors, and maturity factors constructs, i.e., ease-of-use variables derived from the TAM. Liang et $\mathrm{al}^{51}$ examined whether TAM can be applied to explain physician acceptance of computerized physician order entry (CPOE), and found that data analysis provided support for all relationships predicted by TAM but failed to support the relationship between ease of use and attitude. A follow-up analysis showed that this relationship is moderated by CPOE experience (more details of the nine studies are shown in -Table 7).

\section{Discussion}

The review showed that the TAM initially was applied to task-related ICT systems such as EHRs. These were often connected to educational processes leading to that system's impacts on learning and competence were natural critical influences on use intentions. Since the purpose of taskrelated systems is to enhance the users' task performance and improve efficiency, educational concepts can be expected to continue to play a dominant role within TAM in this domain. In other words, for the task-related systems such as EHRs, PU and self-efficacy related to learning can be expected to have stronger effects on usage than PEOU, ${ }^{33}$ i.e., clinical users are likely to accept a new technology mainly if they recognize that it can help them to improve their work performance and build efficacy. ${ }^{52}$ In addition to PU and selfefficacy, system quality, information quality, physicians' autonomy, security and privacy concerns, and cultural and organizational characteristics were found to be important for adoption of task-related technologies, such as EHRs and HISs.

The second aggregation of TAM research was focused on communication systems and telemedicine. The rapid development of worldwide Internet infrastructures has facilitated development of systems in this domain. Telemedicine applications have in particular allowed to introduce new organizational structures in health services ${ }^{40}$ and consequently led to an interest in the use of the TAM to facilitate the organizational adaptation. Health care policy makers are still debating why institutionalizing telemedicine applications on a large scale has been so difficult, ${ }^{53}$ and why health care 


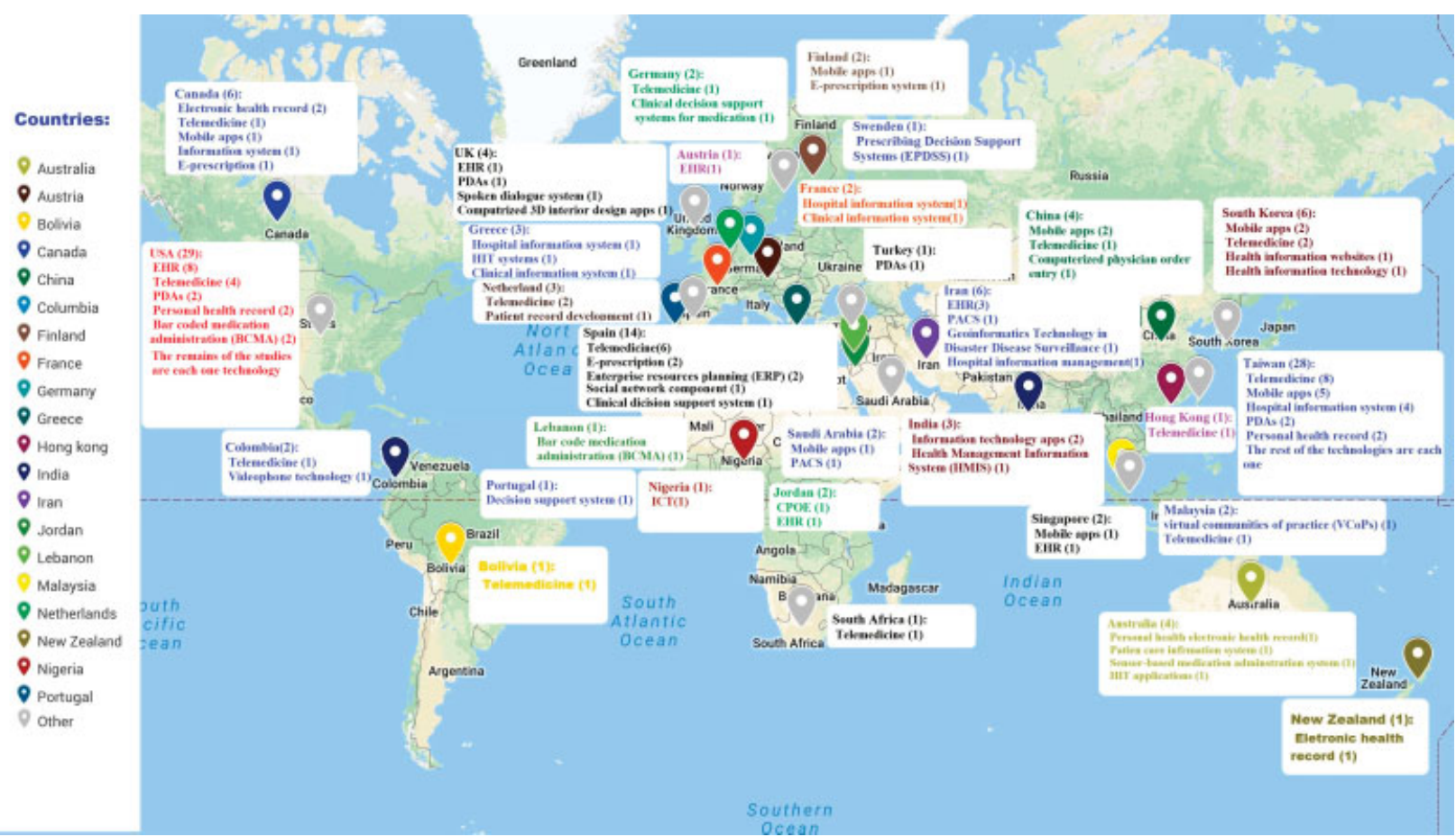

Fig. 4 Technological contexts in using the technology acceptance model between geographical contexts. The parenthesized value is number of studies.

professionals are often averse or indifferent to telemedicine applications. ${ }^{40,54}$ We believe that user rejection is one of the important factors in institutionalizing various types of telemedicine applications. Therefore, it is important to examine the effective factors in accepting telemedicine applications by health care professionals. Consequently, when using the TAM on this category of systems, the validity of analyses with regard to the organizational fit of the novel ICT application is central. ${ }^{55,56}$ Other factors commonly associated with technology adoption in this context include subjective norm, security and confidentiality, facilitators, accessibility, and self-efficacy.

Finally, the most recent trend in TAM use-on mobile technologies-is characterized by involving also patients as users. In this setting, the notion of "hedonic" system aspects, denoting factors associated with pleasure or happiness is of

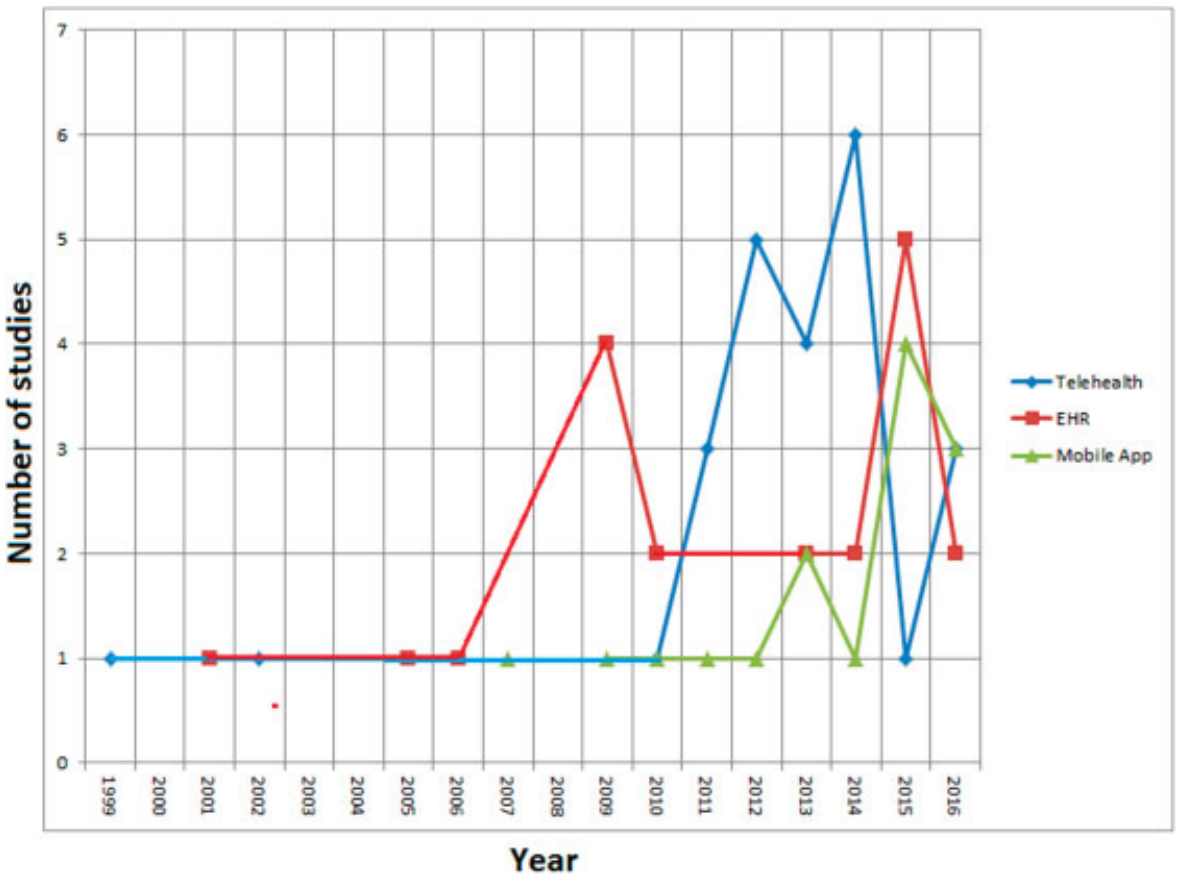

Fig. 5 Distribution of three main technological contexts in using the technology acceptance model by year. 
Table 3 Study user group definitions and the number of studies for each user group

\begin{tabular}{|l|l|}
\hline User groups & $\begin{array}{l}\text { Number of studies, } \\
\text { percentage (\%) }\end{array}$ \\
\hline Physicians & $43(31.8)$ \\
\hline Nurses & $34(25.1)$ \\
\hline Patients & $17(13)$ \\
\hline Health care professionals & $15(11.1)$ \\
\hline Health service staff & $13(9.6)$ \\
\hline General population & $9(6.6)$ \\
\hline Technology users & $8(5.9)$ \\
\hline Managers and providers & $4(2.9)$ \\
\hline Students & $3(2.2)$ \\
\hline Pharmacists & $2(1.4)$ \\
\hline $\begin{array}{l}\text { Physiotherapists and midwives } \\
\text { each }\end{array}$ & $1(0.7)$ \\
\hline
\end{tabular}

importance. ${ }^{57}$ Different from the task-related systems, the concept of hedonic systems focuses on the enjoyable aspect of ICT use and consequently requires other types of factors and variables for analyses of use intentions. Intrinsic motivational factors such as usability and perceived liveliness are in this setting as influential as the PU. The progress from EHRs to mobile technologies in ICT applications has required also the TAM to be dynamically adapted. Based on this, progress of technology introduction in health services cannot be seen to decrease, and a need to modify the TAM to keep up with the new application areas can be also foreseen in the future. Common factors for hedonic such as mobile apps include usability, user satisfaction, reliability, privacy, compatibility, innovativeness, subjective norm, self-efficacy, technical support and training, anxiety, and communication. Also, a theory that integrates with the original TAM to examine the hedonic systems is the self-determination theory (SDT). SDT is a theory of motivation that is concerned with supporting our natural or intrinsic tendencies to behave in effective and healthy ways. ${ }^{58}$

In the extensions of the TAM observed in the review, a wide range of technological context factors and circumstances were

Table 4 Publications addressing the original TAM

\begin{tabular}{|c|c|c|c|c|c|c|}
\hline Author(s) & $\begin{array}{l}\text { Technology } \\
\text { studied/Platform }\end{array}$ & Objective & Year & $\begin{array}{l}\text { Sample } \\
\text { population } \\
\text { and approved } \\
\text { factors }\end{array}$ & Setting & Country \\
\hline Hu et $\mathrm{al}^{42}$ & Telemedicine & $\begin{array}{l}\text { The applicability of the } \\
\text { TAM in explaining } \\
\text { physicians' decisions to } \\
\text { accept telemedicine } \\
\text { technology in the health } \\
\text { care context }\end{array}$ & 1999 & $\begin{array}{l}\text { Physicians } \\
N=421 / \\
\text { perceived ease } \\
\text { of use not } \\
\text { approved }\end{array}$ & Hospital & Hong Kong \\
\hline Barker et al ${ }^{61}$ & $\begin{array}{l}\text { Spoken dialogue } \\
\text { system (SDS) }\end{array}$ & $\begin{array}{l}\text { The application of TAM, to } \\
\text { use spoken dialogue } \\
\text { technology for recording } \\
\text { clinical observations } \\
\text { during an endoscopic } \\
\text { examination }\end{array}$ & 2003 & $\begin{array}{l}\text { Clinicians } \\
(N=12)\end{array}$ & $\begin{array}{l}\text { Endoscopy } \\
\text { center }\end{array}$ & $\begin{array}{l}\text { United Kingd- } \\
\text { om }\end{array}$ \\
\hline Chang et al ${ }^{62}$ & $\begin{array}{l}\text { Triage-based } \\
\text { emergency } \\
\text { medical service } \\
\text { (EMS) personal } \\
\text { digital assistant } \\
\text { (PDA) support } \\
\text { systems }\end{array}$ & $\begin{array}{l}\text { Developing triage-based } \\
\text { EMS (PDA) support } \\
\text { systems among nurses } \\
\text { and physicians by TAM }\end{array}$ & 2004 & $\begin{array}{l}\text { Physicians, } \\
\text { nurses } \\
(N=29)\end{array}$ & $\begin{array}{l}\text { Emergency } \\
\text { medical } \\
\text { center }\end{array}$ & Taiwan \\
\hline Chang et al ${ }^{63}$ & $\begin{array}{l}\text { Emergency } \\
\text { medical service } \\
\text { PDA support } \\
\text { systems }\end{array}$ & $\begin{array}{l}\text { Extending well- } \\
\text { developed, triage-based, } \\
\text { EMS (PDA) support } \\
\text { systems to cover } \\
\text { prehospital emergency } \\
\text { medical services }\end{array}$ & 2004 & $\begin{array}{l}\text { Physicians, } \\
\text { nurses } \\
(N=29)\end{array}$ & Hospital & Taiwan \\
\hline Chen et al ${ }^{64}$ & $\begin{array}{l}\text { Web-based } \\
\text { learning system }\end{array}$ & $\begin{array}{l}\text { Understanding PHNs' BI } \\
\text { toward Web-based } \\
\text { learning based on the } \\
\text { technology acceptance } \\
\text { model (TAM) }\end{array}$ & 2008 & $\begin{array}{l}\text { Nurses } \\
(N=202)\end{array}$ & $\begin{array}{l}\text { Health } \\
\text { centers }\end{array}$ & Taiwan \\
\hline
\end{tabular}


Table 4 (Continued)

\begin{tabular}{|c|c|c|c|c|c|c|}
\hline Author(s) & $\begin{array}{l}\text { Technology } \\
\text { studied/Platform }\end{array}$ & Objective & Year & $\begin{array}{l}\text { Sample } \\
\text { population } \\
\text { and approved } \\
\text { factors }\end{array}$ & Setting & Country \\
\hline Wilkins ${ }^{65}$ & $\begin{array}{l}\text { Electronic health } \\
\text { records (EHR) }\end{array}$ & $\begin{array}{l}\text { Examining factors that } \\
\text { may influence the } \\
\text { adoption of electronic } \\
\text { health records by TAM }\end{array}$ & 2009 & $\begin{array}{l}\text { Health } \\
\text { information } \\
\text { managers } \\
(N=94) \\
\end{array}$ & Hospital & United States \\
\hline Marini et al ${ }^{66}$ & BCMA system & $\begin{array}{l}\text { Using the TAM to } \\
\text { determine the level of } \\
\text { nurses' readiness to use IT } \\
\text { for medication } \\
\text { administration }\end{array}$ & 2009 & $\begin{array}{l}\text { Nurses } \\
(N=276)\end{array}$ & Hospital & Lebanon \\
\hline $\begin{array}{l}\text { Van Schaik } \\
\text { et al }\end{array}$ & $\begin{array}{l}\text { Portable system } \\
\text { for postural } \\
\text { assessment }\end{array}$ & $\begin{array}{l}\text { Assessing the TAM for the } \\
\text { new system }\end{array}$ & 2002 & $\begin{array}{l}\text { Physiothera- } \\
\text { pists } \\
(N=49)\end{array}$ & Spinal unit & $\begin{array}{l}\text { United Kingd- } \\
\text { om }\end{array}$ \\
\hline Huser et al ${ }^{68}$ & $\begin{array}{l}\text { A prototype of a } \\
\text { flowchart-based } \\
\text { analytical } \\
\text { framework } \\
\text { (RetroGuide) }\end{array}$ & $\begin{array}{l}\text { Exploring acceptance of } \\
\text { query systems called } \\
\text { RetroGuide for retrieval } \\
\text { EHR data }\end{array}$ & 2010 & $\begin{array}{l}\text { Human } \\
\text { subjects } \\
(N=18)\end{array}$ & Laboratory & United States \\
\hline Cranen et al $^{69}$ & $\begin{array}{l}\text { Web-based } \\
\text { telemedicine } \\
\text { service }\end{array}$ & $\begin{array}{l}\text { The patients' perceptions } \\
\text { regarding a Web-based } \\
\text { telemedicine service with } \\
\text { TAM among patient }\end{array}$ & 2011 & $\begin{array}{l}\text { Patients } \\
(N=30)\end{array}$ & Homecare & $\begin{array}{l}\text { The } \\
\text { Netherlands }\end{array}$ \\
\hline $\begin{array}{l}\text { Hung and } \\
\text { Jen }\end{array}$ & $\begin{array}{l}\text { Mobile health } \\
\text { management } \\
\text { services (MHMS) }\end{array}$ & $\begin{array}{l}\text { This study introduces } \\
\text { MHMS and employs the } \\
\text { TAM to explore the } \\
\text { intention of students in } \\
\text { Executive Master of } \\
\text { Business Management } \\
\text { programs to adopt mobile } \\
\text { health management } \\
\text { technology }\end{array}$ & 2012 & $\begin{array}{l}\text { Students } \\
(N=170)\end{array}$ & University & Taiwan \\
\hline Aldosari $^{71}$ & $\begin{array}{l}\text { Picture archiving } \\
\text { and } \\
\text { communication } \\
\text { system (PACS) }\end{array}$ & $\begin{array}{l}\text { The TAM was used to } \\
\text { assess the level of } \\
\text { acceptance of the host } \\
\text { PACS by staff in the } \\
\text { radiology department }\end{array}$ & 2012 & $\begin{array}{l}\text { Staffs } \\
(N=89)\end{array}$ & $\begin{array}{l}\text { Radiology } \\
\text { department }\end{array}$ & Saudi Arabia \\
\hline Noblin et $\mathrm{al}^{72}$ & $\begin{array}{l}\text { Personal health } \\
\text { record }\end{array}$ & $\begin{array}{l}\text { The TAM was used to } \\
\text { evaluate to adopt } \\
\text { personal health record }\end{array}$ & 2013 & $\begin{array}{l}\text { Patients } \\
(N=10)\end{array}$ & Hospital & United States \\
\hline $\begin{array}{l}\text { Martínez- } \\
\text { García et al }\end{array}$ & $\begin{array}{l}\text { Social network } \\
\text { component }\end{array}$ & $\begin{array}{l}\text { Assessing acceptance and } \\
\text { use of the social network } \\
\text { component (web 2.0) to } \\
\text { enable the adoption of } \\
\text { shared decisions among } \\
\text { health professionals (this } \\
\text { is highly relevant for } \\
\text { multimorbidity patients } \\
\text { care) using TAM }\end{array}$ & 2013 & $\begin{array}{l}\text { Health care } \\
\text { professionals } \\
(N=10)\end{array}$ & $\begin{array}{l}\text { Health care } \\
\text { center }\end{array}$ & Spain \\
\hline $\begin{array}{l}\text { Monthuy- } \\
\text { Blanc et al }\end{array}$ & $\begin{array}{l}\text { Telemental } \\
\text { health } \\
\text { (psychotherapy } \\
\text { delivered via } \\
\text { videoconferen- } \\
\text { cing) }\end{array}$ & $\begin{array}{l}\text { Understanding the role of } \\
\text { mental health service } \\
\text { providers' attitudes and } \\
\text { perceptions of } \\
\text { psychotherapy delivered } \\
\text { via videoconferencing on } \\
\text { their intention to use this } \\
\text { technology with their } \\
\text { patients }\end{array}$ & 2013 & $\begin{array}{l}\text { Providers of } \\
\text { health care } \\
(N=205)\end{array}$ & $\begin{array}{l}\text { Center of } \\
\text { Telemental }\end{array}$ & Canada \\
\hline
\end{tabular}




\begin{tabular}{|c|c|c|c|c|c|c|}
\hline Author(s) & $\begin{array}{l}\text { Technology } \\
\text { studied/Platform }\end{array}$ & Objective & Year & $\begin{array}{l}\text { Sample } \\
\text { population } \\
\text { and approved } \\
\text { factors }\end{array}$ & Setting & Country \\
\hline $\begin{array}{l}\text { Abdekhoda } \\
\text { et } a^{75}\end{array}$ & $\begin{array}{l}\text { Health } \\
\text { information } \\
\text { management } \\
\text { system }\end{array}$ & $\begin{array}{l}\text { The acceptance of } \\
\text { information technology in } \\
\text { the context of health } \\
\text { information management } \\
\text { (HIM) by utilizing TAM }\end{array}$ & 2014 & $\begin{array}{l}\text { Worker of } \\
\text { medical record } \\
(N=187)\end{array}$ & Hospital & Iran \\
\hline $\begin{array}{l}\text { Cilliers and } \\
\text { Stephen }\end{array}$ & Telemedicine & $\begin{array}{l}\text { Using of the TAM to } \\
\text { identify the factors that } \\
\text { influence the user } \\
\text { acceptance of } \\
\text { telemedicine among } \\
\text { health care workers }\end{array}$ & 2014 & $\begin{array}{l}\text { Health care } \\
\text { workers } \\
(n=75)\end{array}$ & $\begin{array}{l}\text { Hospital and } \\
\text { clinic }\end{array}$ & South Africa \\
\hline $\begin{array}{l}\text { Ologeanu- } \\
\text { Taddei et al }^{77}\end{array}$ & $\begin{array}{l}\text { Hospital } \\
\text { information } \\
\text { system (HIS) }\end{array}$ & $\begin{array}{l}\text { Examining key factors of a } \\
\text { HIS acceptance for the } \\
\text { care staff, based on the } \\
\text { main concepts of TAM }\end{array}$ & 2015 & $\begin{array}{l}\text { Staffs } \\
(N=1,942)\end{array}$ & Hospital & France \\
\hline Money et $\mathrm{al}^{78}$ & $\begin{array}{l}\text { Computerized 3D } \\
\text { interior design } \\
\text { applications } \\
\text { (CIDAs) }\end{array}$ & $\begin{array}{l}\text { Exploring the perceptions } \\
\text { of community dwelling } \\
\text { older adults with regards } \\
\text { to adopting and using } \\
\text { CIDAs with TAM }\end{array}$ & 2015 & $\begin{array}{l}\text { Older adult } \\
(N=10)\end{array}$ & Homecare & $\begin{array}{l}\text { United Kingd- } \\
\text { om }\end{array}$ \\
\hline $\begin{array}{l}\text { Faruque } \\
\text { et al }\end{array}$ & $\begin{array}{l}\text { Geoinformatics } \\
\text { technology in } \\
\text { disaster disease } \\
\text { surveillance }\end{array}$ & $\begin{array}{l}\text { Assessing the feasibility of } \\
\text { using geoinformatics } \\
\text { technology in disaster } \\
\text { disease surveillance uses } \\
\text { by self-administration } \\
\text { based on the technology } \\
\text { acceptance model (TAM) }\end{array}$ & 2015 & $\begin{array}{l}\text { Personnel } \\
(N=50)\end{array}$ & $\begin{array}{l}\text { Health } \\
\text { centers }\end{array}$ & Iran \\
\hline $\begin{array}{l}\text { Kivekäs } \\
\text { et al }\end{array}$ & $\begin{array}{l}\text { Electronic } \\
\text { prescription } \\
\text { (e-prescription) } \\
\text { system }\end{array}$ & $\begin{array}{l}\text { Assessing general } \\
\text { practitioners' (GP) } \\
\text { experience of an } \\
\text { electronic prescription (e- } \\
\text { prescription) system and } \\
\text { the use of a national } \\
\text { prescription center }\end{array}$ & 2016 & $\begin{array}{l}\text { General } \\
\text { practitioners } \\
(N=269)\end{array}$ & Hospital & Finland \\
\hline $\begin{array}{l}\text { Abdullah } \\
\text { et } \mathrm{al}^{81}\end{array}$ & $\begin{array}{l}\text { Telemonitoring } \\
\text { of home blood } \\
\text { pressure (BP) }\end{array}$ & $\begin{array}{l}\text { Exploring patients' } \\
\text { acceptance of a BP } \\
\text { telemonitoring service } \\
\text { delivered in primary care } \\
\text { based on the technology } \\
\text { acceptance model (TAM) }\end{array}$ & 2016 & $\begin{array}{l}\text { Patients } \\
(N=17)\end{array}$ & Homecare & Malaysia \\
\hline $\begin{array}{l}\text { Hanauer } \\
\text { et al }\end{array}$ & $\begin{array}{l}\text { Computer-based } \\
\text { query } \\
\text { recommendation } \\
\text { algorithm }\end{array}$ & $\begin{array}{l}\text { Assessing computer- } \\
\text { based query } \\
\text { recommendation } \\
\text { algorithm as part of a } \\
\text { search engine that } \\
\text { facilitates retrieval of } \\
\text { information from EHRs } \\
\text { using TAM }\end{array}$ & 2017 & $\begin{array}{l}\text { Clinicians, } \\
\text { staffs } \\
(N=33)\end{array}$ & Hospital & United States \\
\hline
\end{tabular}

Abbreviations: BCMA, bar code medication administration; BI, business intelligence; EHR, electronic health record; IT, information technology; PHN, public health nurse; TAM, technology acceptance model.

introduced. Examples of such factors include physicians' autonomy, doctor-patient relationship, project team competency, clinical safely, job fit, and optimism, as well as patient user group, ${ }^{59}$ voluntariness of the ICT use, and whether the ICT systems were prototypes, trial systems, to-be-implemented systems, or implemented systems. Other revisions had more to do with explicitly stating contextual circumstances, rather than extensions per se. For instance, over the life course of an ICT application, the relationships in the TAM may change, e.g., usability may initially be critical but less important later on. Two methods to add novel concepts and variables to the TAM were highlighted in this review. The first, theory-based 
614 A Systematic Review of the Technology Acceptance Model Rahimi et al.

Table 5 Publications addressing extension and development of TAM

\begin{tabular}{|c|c|c|c|c|c|c|}
\hline Author(s) & $\begin{array}{l}\text { Technology } \\
\text { studied }\end{array}$ & Main topic & Years & Sample & $\begin{array}{l}\text { Setting/Incorporated } \\
\text { theories and variable } \\
\text { with the TAM }\end{array}$ & Country \\
\hline $\begin{array}{l}\text { Rawstorne } \\
\text { et al }{ }^{83}\end{array}$ & $\begin{array}{l}\text { Patient care } \\
\text { information } \\
\text { system }\end{array}$ & $\begin{array}{l}\text { Identifying the relevant } \\
\text { issues necessary for } \\
\text { applying the } \\
\text { technology acceptance } \\
\text { model and the theory } \\
\text { of planned behavior to } \\
\text { the prediction and } \\
\text { explanation of } \\
\text { mandated } \\
\text { IS usage }\end{array}$ & 2000 & $\begin{array}{l}\text { Nurses } \\
(N=61)\end{array}$ & $\begin{array}{l}\text { Hospital/theory of } \\
\text { planned behavior (TPB) }\end{array}$ & Australia \\
\hline $\begin{array}{l}\text { Handy } \\
\text { et al }\end{array}$ & $\begin{array}{l}\text { Electronic } \\
\text { medical } \\
\text { records (EMR) }\end{array}$ & $\begin{array}{l}\text { Studying primary care } \\
\text { practitioners' views of } \\
\text { an electronic medical } \\
\text { records (EMR) system } \\
\text { for maternity patients }\end{array}$ & 2001 & $\begin{array}{l}\text { Physicians } \\
\text { and } \\
\text { midwives } \\
(N=167)\end{array}$ & $\begin{array}{l}\text { Hospital/System } \\
\text { acceptability, system } \\
\text { characteristics, } \\
\text { organizational } \\
\text { characteristics, } \\
\text { individual characteristics }\end{array}$ & $\begin{array}{l}\text { New } \\
\text { Zealand }\end{array}$ \\
\hline $\begin{array}{l}\text { Chismar } \\
\text { and } \\
\text { Sonja } 85\end{array}$ & $\begin{array}{l}\text { Internet and } \\
\text { Internet-based } \\
\text { health } \\
\text { applications }\end{array}$ & $\begin{array}{l}\text { Testing the extension } \\
\text { to a widely used model } \\
\text { in the information } \\
\text { systems especially } \\
\text { Internet in pediatrics }\end{array}$ & 2002 & $\begin{array}{l}\text { Pediatri- } \\
\text { cians } \\
(N=89)\end{array}$ & $\begin{array}{l}\text { Hospital/the TAM2 } \\
\text { theory }\end{array}$ & $\begin{array}{l}\text { United } \\
\text { States }\end{array}$ \\
\hline $\begin{array}{l}\text { Liang } \\
\text { et } \mathrm{al}^{86}\end{array}$ & $\begin{array}{l}\text { Personal digital } \\
\text { assistants } \\
\text { (PDAs) }\end{array}$ & $\begin{array}{l}\text { Predicting TAM to } \\
\text { actual PDA usage }\end{array}$ & 2003 & $\begin{array}{l}\text { Health care } \\
\text { profession- } \\
\text { als } \\
(N=173)\end{array}$ & $\begin{array}{l}\text {-/compatibility, support, } \\
\text { personal innovativeness, } \\
\text { job relevance }\end{array}$ & $\begin{array}{l}\text { United } \\
\text { States }\end{array}$ \\
\hline $\begin{array}{l}\text { Liu and } \\
\mathrm{Ma}^{87}\end{array}$ & $\begin{array}{l}\text { Service- } \\
\text { oriented } \\
\text { medical } \\
\text { records }\end{array}$ & $\begin{array}{l}\text { Extending TAM by } \\
\text { embedding perceived } \\
\text { service level (PSL) as a } \\
\text { causal antecedent for } \\
\text { health care workers' } \\
\text { willingness to use } \\
\text { application service- } \\
\text { oriented medical } \\
\text { records }\end{array}$ & 2005 & $\begin{array}{l}\text { Health care } \\
\text { worker } \\
(N=79)\end{array}$ & $\begin{array}{l}\text { Hospital/Perceived } \\
\text { service level }\end{array}$ & $\begin{array}{l}\text { United } \\
\text { States }\end{array}$ \\
\hline Han et $\mathrm{al}^{43}$ & Mobile system & $\begin{array}{l}\text { Examining acceptance } \\
\text { of mobile system } \\
\text { among physicians with } \\
\text { the aid from mainly } \\
\text { TAM, UTAUT and } \\
\text { Personal } \\
\text { Innovativeness in the } \\
\text { Domain of Information } \\
\text { Technology (PIIT) } \\
\text { models }\end{array}$ & 2006 & $\begin{array}{l}\text { Physicians } \\
(N=151)\end{array}$ & $\begin{array}{l}\text { Health care sector/ } \\
\text { gender, experience, age, } \\
\text { personal innovativeness, } \\
\text { compatibility, social } \\
\text { influence }\end{array}$ & Finland \\
\hline $\begin{array}{l}\text { Liu and } \\
\mathrm{Ma}^{88}\end{array}$ & $\begin{array}{l}\text { Electronic } \\
\text { medical } \\
\text { records (EMR) }\end{array}$ & $\begin{array}{l}\text { Introducing the notion } \\
\text { of perceived } \\
\text { system performance } \\
\text { (PSP) to extend the } \\
\text { TAM }\end{array}$ & 2006 & $\begin{array}{l}\text { Medical } \\
\text { profession- } \\
\text { als } \\
(N=77)\end{array}$ & $\begin{array}{l}\text { Hospital/Perceived } \\
\text { system performance }\end{array}$ & $\begin{array}{l}\text { United } \\
\text { States }\end{array}$ \\
\hline Palm et al ${ }^{89}$ & $\begin{array}{l}\text { Clinical } \\
\text { information } \\
\text { system (CIS) }\end{array}$ & $\begin{array}{l}\text { Designing an electronic } \\
\text { survey instrument from } \\
\text { two theoretical models } \\
\text { (Delone and McLean, } \\
\text { and TAM) to assess the } \\
\text { acceptability of an } \\
\text { integrated CIS }\end{array}$ & 2006 & $\begin{array}{l}\text { Physicians, } \\
\text { nurses, } \\
\text { and } \\
\text { secretaries } \\
(N=324)\end{array}$ & $\begin{array}{l}\text { Hospital/Building on the } \\
\text { TAM and the DeLone and } \\
\text { McLean ISS models }\end{array}$ & France \\
\hline $\begin{array}{l}\text { Kim and } \\
\text { Chang }\end{array}$ & & $\begin{array}{l}\text { Identifying the core } \\
\text { functional factors in }\end{array}$ & 2007 & $\begin{array}{l}\text { Users } \\
(N=228)\end{array}$ & $\begin{array}{l}\text { Home/Information } \\
\text { search, usage support, }\end{array}$ & $\begin{array}{l}\text { South } \\
\text { Korea }\end{array}$ \\
\hline
\end{tabular}




\begin{tabular}{|c|c|c|c|c|c|c|}
\hline Author(s) & $\begin{array}{l}\text { Technology } \\
\text { studied }\end{array}$ & Main topic & Years & Sample & $\begin{array}{l}\text { Setting/Incorporated } \\
\text { theories and variable } \\
\text { with the TAM }\end{array}$ & Country \\
\hline & $\begin{array}{l}\text { Health } \\
\text { information } \\
\text { Web sites }\end{array}$ & $\begin{array}{l}\text { designing and } \\
\text { operating health } \\
\text { information Web sites }\end{array}$ & & & $\begin{array}{l}\text { customization, } \\
\text { purchase, and security }\end{array}$ & \\
\hline Wu et $\mathrm{al}^{91}$ & $\begin{array}{l}\text { Mobile health } \\
\text { care systems }\end{array}$ & $\begin{array}{l}\text { Examining determines } \\
\text { mobile health care } \\
\text { systems (MHS) } \\
\text { acceptance by health } \\
\text { care professionals } \\
\text { based on revised TAM }\end{array}$ & 2007 & $\begin{array}{l}\text { Physicians, } \\
\text { nurses, and } \\
\text { medical } \\
\text { technicians } \\
(N=137)\end{array}$ & $\begin{array}{l}\text { Hospital/MHS self- } \\
\text { efficacy, technical } \\
\text { support and training, } \\
\text { compatibility }\end{array}$ & Taiwan \\
\hline Tung et al ${ }^{92}$ & $\begin{array}{l}\text { Electronic } \\
\text { logistics } \\
\text { information } \\
\text { system }\end{array}$ & $\begin{array}{l}\text { Nurses' acceptance of } \\
\text { the electronic logistics } \\
\text { information system } \\
\text { with new hybrid TAM }\end{array}$ & 2008 & $\begin{array}{l}\text { Nurses } \\
(N=258)\end{array}$ & $\begin{array}{l}\text { Hospital/Perceived } \\
\text { financial cost, } \\
\text { compatibility, trust }\end{array}$ & Taiwan \\
\hline Lai et $\mathrm{al}^{93}$ & $\begin{array}{l}\text { Tailored } \\
\text { Interventions } \\
\text { for } \\
\text { management } \\
\text { of DEpressive } \\
\text { Symptoms } \\
\text { (TIDES) }\end{array}$ & $\begin{array}{l}\text { Designing Tailored } \\
\text { Interventions for } \\
\text { management of } \\
\text { DEpressive Symptoms } \\
\text { (TIDES) program based } \\
\text { on an extension of the } \\
\text { TAM }\end{array}$ & 2008 & $\begin{array}{l}\text { Patients } \\
(N=32)\end{array}$ & $\begin{array}{l}\text { Clinics/framework based } \\
\text { on TAM2 (subjective } \\
\text { norm, job relevance, } \\
\text { experience) and modified } \\
\text { TAM (socio-demo, } \\
\text { adjustment, job } \\
\text { relevance) }\end{array}$ & $\begin{array}{l}\text { United } \\
\text { States }\end{array}$ \\
\hline Wu et $\mathrm{al}^{94}$ & $\begin{array}{l}\text { Adverse event } \\
\text { reporting } \\
\text { system }\end{array}$ & $\begin{array}{l}\text { Investigating } \\
\text { determines acceptance } \\
\text { of adverse event } \\
\text { reporting systems by } \\
\text { health care } \\
\text { professionals with } \\
\text { extending TAM that } \\
\text { integrates variables } \\
\text { connoting trust and } \\
\text { management support } \\
\text { into the model }\end{array}$ & 2008 & $\begin{array}{l}\text { Health care } \\
\text { profession- } \\
\text { als } \\
(N=290)\end{array}$ & $\begin{array}{l}\text { Hospital/trust, } \\
\text { management support, } \\
\text { subjective norm }\end{array}$ & Taiwan \\
\hline Yu et $\mathrm{al}^{95}$ & $\begin{array}{l}\text { Health } \\
\text { information } \\
\text { technology } \\
\text { applications }\end{array}$ & $\begin{array}{l}\text { Applying a modified } \\
\text { version of the TAM2 to } \\
\text { examine the factors } \\
\text { determining the } \\
\text { acceptance of health IT } \\
\text { applications }\end{array}$ & 2009 & $\begin{array}{l}\text { Staff } \\
\text { members } \\
\text { from long- } \\
\text { term care } \\
\text { facilities } \\
(N=134)\end{array}$ & $\begin{array}{l}\text { Long-term care/age, } \\
\text { subjective norm, image, } \\
\text { job level, work } \\
\text { experience, computer } \\
\text { skills, voluntariness }\end{array}$ & Australia \\
\hline $\begin{array}{l}\text { Dasgupta } \\
\text { et al }\end{array}$ & $\begin{array}{l}\text { Personal digital } \\
\text { assistants } \\
\text { (PDAs) }\end{array}$ & $\begin{array}{l}\text { Evaluating } \\
\text { pharmacists' } \\
\text { behavioral intention to } \\
\text { use PDAs with TAM2 }\end{array}$ & 2009 & $\begin{array}{l}\text { Pharma- } \\
\text { cists } \\
(N=295)\end{array}$ & $\begin{array}{l}\text { Hospital and } \\
\text { community } \\
\text { pharmacies/The TAM2 } \\
\text { theory }\end{array}$ & $\begin{array}{l}\text { United } \\
\text { States }\end{array}$ \\
\hline Ilie et al $^{97}$ & $\begin{array}{l}\text { Electronic } \\
\text { medical record } \\
(\text { EMR) }\end{array}$ & $\begin{array}{l}\text { Examining physicians' } \\
\text { responses to uses of } \\
\text { EMR bases on TAM }\end{array}$ & 2009 & $\begin{array}{l}\text { Physicians } \\
(N=199)\end{array}$ & $\begin{array}{l}\text { Hospital/System } \\
\text { accessibility }\end{array}$ & $\begin{array}{l}\text { United } \\
\text { States }\end{array}$ \\
\hline $\begin{array}{l}\text { Trimmer } \\
\text { et al }\end{array}$ & $\begin{array}{l}\text { Electronic } \\
\text { medical } \\
\text { records (EMRs) }\end{array}$ & $\begin{array}{l}\text { Application models } \\
\text { TAM, UTAUT, and } \\
\text { organizational culture } \\
\text { in several different } \\
\text { phase for acceptance } \\
\text { EMR }\end{array}$ & 2009 & $\begin{array}{l}\text { Physicians } \\
(N=-)\end{array}$ & $\begin{array}{l}\text { Residency in family } \\
\text { medicine/Derived from } \\
\text { TAM, UTAUT, and } \\
\text { organizational culture }\end{array}$ & $\begin{array}{l}\text { United } \\
\text { States }\end{array}$ \\
\hline $\begin{array}{l}\text { Lin and } \\
\text { Yang } 99\end{array}$ & $\begin{array}{l}\text { Asthma care } \\
\text { mobile service } \\
\text { (ACMS) }= \\
\text { mobile phone }\end{array}$ & $\begin{array}{l}\text { Integrating TAM and } \\
\text { "subjective norm" and } \\
\text { "innovativeness" in } \\
\text { acceptance ACMS }\end{array}$ & 2009 & $\begin{array}{l}\text { Patients } \\
(N=229)\end{array}$ & $\begin{array}{l}\text { Remote areas/person- } \\
\text { centered, } \\
\text { communication }\end{array}$ & China \\
\hline $\begin{array}{l}\text { Aggelidis } \\
\text { and }\end{array}$ & $\begin{array}{l}\text { Hospital } \\
\text { information } \\
\text { system (HIS) }\end{array}$ & $\begin{array}{l}\text { Examining HIS } \\
\text { acceptance by hospital }\end{array}$ & 2009 & $\begin{array}{l}\text { Hospital } \\
\text { personnel } \\
(N=283)\end{array}$ & $\begin{array}{l}\text { Hospital/Derived based } \\
\text { on UTAUT and TAM } \\
\text { (Compatibility, training, }\end{array}$ & Greece \\
\hline
\end{tabular}


Table 5 (Continued)

\begin{tabular}{|c|c|c|c|c|c|c|}
\hline Author(s) & $\begin{array}{l}\text { Technology } \\
\text { studied }\end{array}$ & Main topic & Years & Sample & $\begin{array}{l}\text { Setting/Incorporated } \\
\text { theories and variable } \\
\text { with the TAM }\end{array}$ & Country \\
\hline $\begin{array}{l}\text { Chatzo- } \\
\text { glou }^{100}\end{array}$ & & $\begin{array}{l}\text { personnel bases on } \\
\text { TAM }\end{array}$ & & & $\begin{array}{l}\text { social influence, } \\
\text { facilitating condition, } \\
\text { self-efficiency, anxiety) }\end{array}$ & \\
\hline $\begin{array}{l}\text { Hyun } \\
\text { et al }\end{array}$ & $\begin{array}{l}\text { Structured } \\
\text { narrative } \\
\text { electronic } \\
\text { health record } \\
\text { (EHR) model } \\
\text { (electronic } \\
\text { nursing } \\
\text { documenta- } \\
\text { tion system) }\end{array}$ & $\begin{array}{l}\text { Applying theory-based } \\
\text { (combined technology } \\
\text { acceptance model and } \\
\text { task-technology fit } \\
\text { model) and user- } \\
\text { centered methods to } \\
\text { explore nurses' } \\
\text { perceptions of } \\
\text { functional } \\
\text { requirements for an } \\
\text { electronic nursing } \\
\text { documentation system }\end{array}$ & 2009 & $\begin{array}{l}\text { Nurses } \\
(N=17)\end{array}$ & $\begin{array}{l}\text { Hospital/Combined TAM } \\
\text { and task-technology fit } \\
\text { (TTF) model }\end{array}$ & $\begin{array}{l}\text { United } \\
\text { States }\end{array}$ \\
\hline $\begin{array}{l}\text { Vishwa- } \\
\text { nath } \\
\text { et al }\end{array}$ & $\begin{array}{l}\text { Personal digital } \\
\text { assistant (PDA) }\end{array}$ & $\begin{array}{l}\text { Exploring the } \\
\text { determinants of } \\
\text { personal digital } \\
\text { assistant (PDA) } \\
\text { adoption in health care } \\
\text { with TAM }\end{array}$ & 2009 & $\begin{array}{l}\text { Physicians } \\
(N=215)\end{array}$ & $\begin{array}{l}\text { Hospital/age, position in } \\
\text { hospital, cluster } \\
\text { ownership, specialty }\end{array}$ & $\begin{array}{l}\text { United } \\
\text { States }\end{array}$ \\
\hline $\begin{array}{l}\text { Morton } \\
\text { and } \\
\text { Susan }\end{array}$ & $\begin{array}{l}\text { Electronic } \\
\text { health record } \\
(\text { EHR) }\end{array}$ & $\begin{array}{l}\text { Adopting of an } \\
\text { interoperable EHR in } \\
\text { ambulatory card uses } \\
\text { innovation diffusion } \\
\text { theory and the TAM }\end{array}$ & 2010 & $\begin{array}{l}\text { Physicians } \\
(N=802)\end{array}$ & $\begin{array}{l}\text { University/Combining } \\
\text { innovation diffusion } \\
\text { theory (IDT) and the TAM }\end{array}$ & $\begin{array}{l}\text { United } \\
\text { States }\end{array}$ \\
\hline $\begin{array}{l}\text { Zhang } \\
\text { et al }\end{array}$ & $\begin{array}{l}\text { Mobile } \\
\text { homecare } \\
\text { nursing }\end{array}$ & $\begin{array}{l}\text { Applying TAM2 in } \\
\text { mobile homecare } \\
\text { nursing }\end{array}$ & 2010 & $\begin{array}{l}\text { Nurses } \\
(N=91)\end{array}$ & Home/The TAM2 theory & Canada \\
\hline Stocker $^{105}$ & $\begin{array}{l}\text { Electronic } \\
\text { medical } \\
\text { records (EMRs) }\end{array}$ & $\begin{array}{l}\text { Evaluating the TAM } \\
\text { relevance of the } \\
\text { intention of nurses to } \\
\text { use electronic medical } \\
\text { records in acute health } \\
\text { care settings }\end{array}$ & 2010 & $\begin{array}{l}\text { Nurses } \\
(N=97)\end{array}$ & $\begin{array}{l}\text { Hospital/Environment or } \\
\text { context, nurse } \\
\text { characteristics, EHR } \\
\text { characteristic }\end{array}$ & $\begin{array}{l}\text { United } \\
\text { States }\end{array}$ \\
\hline Lim et al ${ }^{106}$ & Mobile phones & $\begin{array}{l}\text { Women's acceptance } \\
\text { of using mobile phones } \\
\text { to seek health } \\
\text { information basis on } \\
\text { TAM }\end{array}$ & 2011 & $\begin{array}{l}\text { Women } \\
(N=175)\end{array}$ & $\begin{array}{l}\text { Home care/Self-efficacy, } \\
\text { anxiety, prior experience }\end{array}$ & Singapore \\
\hline $\begin{array}{l}\text { Schnall and } \\
\text { Bakken } 107\end{array}$ & $\begin{array}{l}\text { Continuity of } \\
\text { care record } \\
\text { (CCR) }\end{array}$ & $\begin{array}{l}\text { Assessing the } \\
\text { applicability of TAM } \\
\text { constructs in explaining } \\
\text { HIV case managers' } \\
\text { behavioral intention to } \\
\text { use a CCR }\end{array}$ & 2011 & $\begin{array}{l}\text { Managers } \\
(N=94)\end{array}$ & $\begin{array}{l}\text { Center of HIV care/ } \\
\text { Perceived barriers to use }\end{array}$ & $\begin{array}{l}\text { United } \\
\text { States }\end{array}$ \\
\hline $\begin{array}{l}\text { Kowitlawa- } \\
\text { kul }^{108}\end{array}$ & $\begin{array}{l}\text { Telemedicine/ } \\
\text { electronic or } \\
\text { remote } \\
\text { technology } \\
\text { (elCU) }\end{array}$ & $\begin{array}{l}\text { Determining factors } \\
\text { and predictors that } \\
\text { influence nurses' } \\
\text { intention to use the } \\
\text { eICU technology bases } \\
\text { on TAM }\end{array}$ & 2011 & $\begin{array}{l}\text { Nurses } \\
(N=117)\end{array}$ & $\begin{array}{l}\text { Hospital/Support from } \\
\text { physicians, years } \\
\text { working in the hospital, } \\
\text { support from } \\
\text { administrator }\end{array}$ & $\begin{array}{l}\text { United } \\
\text { States }\end{array}$ \\
\hline $\begin{array}{l}\text { Egea and } \\
\text { Gonzá- } \\
\text { lez } 109\end{array}$ & $\begin{array}{l}\text { Electronic } \\
\text { health care } \\
\text { records (EHCR) }\end{array}$ & $\begin{array}{l}\text { Explaining physicians' } \\
\text { acceptance for } \\
\text { electronic health care } \\
\text { records (EHCR systems) }\end{array}$ & 2011 & $\begin{array}{l}\text { Physicians } \\
(N=254)\end{array}$ & $\begin{array}{l}\text { Hospital/Perceptions of } \\
\text { institutional trust, } \\
\text { perceived risk, } \\
\text { information integrity }\end{array}$ & Spain \\
\hline
\end{tabular}




\begin{tabular}{|c|c|c|c|c|c|c|}
\hline Author(s) & $\begin{array}{l}\text { Technology } \\
\text { studied }\end{array}$ & Main topic & Years & Sample & $\begin{array}{l}\text { Setting/Incorporated } \\
\text { theories and variable } \\
\text { with the TAM }\end{array}$ & Country \\
\hline $\begin{array}{l}\text { Hsiao } \\
\text { et al }\end{array}$ & $\begin{array}{l}\text { Hospital } \\
\text { information } \\
\text { systems (HIS) }\end{array}$ & $\begin{array}{l}\text { The application of TAM } \\
\text { for evaluate HIS in } \\
\text { among nursing } \\
\text { personnel }\end{array}$ & 2011 & $\begin{array}{l}\text { Nurses } \\
(N=501)\end{array}$ & $\begin{array}{l}\text { Hospital/system quality, } \\
\text { information quality, user } \\
\text { self-efficacy, } \\
\text { compatibility, top } \\
\text { management support, } \\
\text { and project team } \\
\text { competency }\end{array}$ & Taiwan \\
\hline $\begin{array}{l}\text { Orruño } \\
\text { et al }\end{array}$ & $\begin{array}{l}\text { Teledermatol- } \\
\text { ogy }\end{array}$ & $\begin{array}{l}\text { Examining intention of } \\
\text { physicians to use } \\
\text { teledermatology using } \\
\text { a modified TAM }\end{array}$ & 2011 & $\begin{array}{l}\text { Physicians } \\
(N=171)\end{array}$ & $\begin{array}{l}\text { Home/Subjective norm, } \\
\text { facilitator, habit, } \\
\text { compatibility }\end{array}$ & Spain \\
\hline $\begin{array}{l}\text { Melas } \\
\text { et al }\end{array}$ & $\begin{array}{l}\text { Clinical } \\
\text { information } \\
\text { systems }\end{array}$ & $\begin{array}{l}\text { Explaining intention to } \\
\text { use clinical information } \\
\text { systems based on TAM }\end{array}$ & 2011 & $\begin{array}{l}\text { Medical } \\
\text { staff (total } \\
{[N=604]} \\
\text { physicians- } \\
=534)\end{array}$ & $\begin{array}{l}\text { Hospital/Physician } \\
\text { specialty, ICT knowledge, } \\
\text { ICT feature demand }\end{array}$ & Greece \\
\hline $\begin{array}{l}\text { Pai and } \\
\mathrm{Kai}^{113}\end{array}$ & $\begin{array}{l}\text { Health care } \\
\text { information } \\
\text { systems }\end{array}$ & $\begin{array}{l}\text { Adopting the system } \\
\text { and services based on } \\
\text { Model proposed by } \\
\text { DeLone and Mclean and } \\
\text { TAM }\end{array}$ & 2011 & $\begin{array}{l}\text { Nurses, } \\
\text { head } \\
\text { directors, } \\
\text { and other } \\
\text { related } \\
\text { personnel } \\
(N=366) \\
\end{array}$ & $\begin{array}{l}\text { Hospital/Model } \\
\text { proposed by DeLone } \\
\text { and Mclean and TAM }\end{array}$ & Taiwan \\
\hline $\begin{array}{l}\text { Jimoh } \\
\text { et al }\end{array}$ & $\begin{array}{l}\text { Information } \\
\text { and } \\
\text { communica- } \\
\text { tion } \\
\text { technology } \\
\text { (ICT) }\end{array}$ & $\begin{array}{l}\text { Using modified TAM in } \\
\text { among maternal and } \\
\text { child health workers }\end{array}$ & 2012 & $\begin{array}{l}\text { Health } \\
\text { workers } \\
(N=200)\end{array}$ & $\begin{array}{l}\text { Rural regions/ } \\
\text { knowledge, endemic } \\
\text { barriers (knowledge a } \\
\text { separate factor from } \\
\text { attitude) }\end{array}$ & Nigeria \\
\hline Lu et $\mathrm{al}^{115}$ & $\begin{array}{l}\text { Hospital } \\
\text { information } \\
\text { system (HIS) }\end{array}$ & $\begin{array}{l}\text { Exploring factors } \\
\text { influencing the } \\
\text { acceptance of HISs by } \\
\text { nurses with derived } \\
\text { model from TAM }\end{array}$ & 2012 & $\begin{array}{l}\text { Nurses } \\
(N=277)\end{array}$ & $\begin{array}{l}\text { Hospital/Information } \\
\text { system success model }\end{array}$ & Taiwan \\
\hline $\begin{array}{l}\text { Lakshmi } \\
\text { and } \\
\text { Rajaram }^{116}\end{array}$ & $\begin{array}{l}\text { Information } \\
\text { technology (IT) } \\
\text { applications } \\
\text { and } \\
\text { innovativeness }\end{array}$ & $\begin{array}{l}\text { Analyzing the influence } \\
\text { of IT applications and } \\
\text { innovativeness on the } \\
\text { acceptance of rural } \\
\text { health care services } \\
\text { uses by TAM }\end{array}$ & 2012 & $\begin{array}{l}\text { Health } \\
\text { personnel } \\
(N=465)\end{array}$ & $\begin{array}{l}\text { Rural centers/ } \\
\text { Information technology } \\
\text { exposure, } \\
\text { innovativeness, online } \\
\text { information dependence }\end{array}$ & India \\
\hline Jian et al ${ }^{117}$ & $\begin{array}{l}\text { USB-based } \\
\text { personal } \\
\text { health records } \\
\text { (PHRs) }\end{array}$ & $\begin{array}{l}\text { Factors that influencing } \\
\text { consumer adoption of } \\
\text { USB-based personal } \\
\text { health records by TAM }\end{array}$ & 2012 & $\begin{array}{l}\text { Patients } \\
(N=- \\
1,465)\end{array}$ & $\begin{array}{l}\text { Hospital/Subjective } \\
\text { norm }\end{array}$ & Taiwan \\
\hline $\begin{array}{l}\text { Escobar- } \\
\text { Rodríguez } \\
\text { et al }\end{array}$ & $\begin{array}{l}\text { e-Prescriptions } \\
\text { and automated } \\
\text { medication } \\
\text { management } \\
\text { systems }\end{array}$ & $\begin{array}{l}\text { Investigating health } \\
\text { care personnel to use e- } \\
\text { prescriptions and } \\
\text { automated medication } \\
\text { management systems } \\
\text { with extensive TAM }\end{array}$ & 2012 & $\begin{array}{l}\text { Physicians, } \\
\text { nurses } \\
(N=209)\end{array}$ & $\begin{array}{l}\text { Hospital/perceived } \\
\text { compatibility, perceived } \\
\text { usefulness to enhance } \\
\text { control systems, } \\
\text { training, perceived risks }\end{array}$ & Spain \\
\hline $\begin{array}{l}\text { Ketikidis } \\
\text { et al }{ }^{119}\end{array}$ & HIT systems & $\begin{array}{l}\text { Applying modified TAM } \\
\text { in acceptance of HIT } \\
\text { systems in health care } \\
\text { personnel }\end{array}$ & 2012 & $\begin{array}{l}\text { Health } \\
\text { profession- } \\
\text { als (nurses } \\
\text { and } \\
\text { medical } \\
\text { doctors) } \\
(N=133)\end{array}$ & $\begin{array}{l}\text { Hospital/Computer } \\
\text { anxiety, relevance, self- } \\
\text { efficacy, subjective and } \\
\text { descriptive norms, } \\
\text { familiarity/ use of } \\
\text { computers }\end{array}$ & Greece \\
\hline
\end{tabular}


618 A Systematic Review of the Technology Acceptance Model Rahimi et al.

Table 5 (Continued)

\begin{tabular}{|c|c|c|c|c|c|c|}
\hline Author(s) & $\begin{array}{l}\text { Technology } \\
\text { studied }\end{array}$ & Main topic & Years & Sample & $\begin{array}{l}\text { Setting/Incorporated } \\
\text { theories and variable } \\
\text { with the TAM }\end{array}$ & Country \\
\hline $\begin{array}{l}\text { Chen and } \\
\text { Hsiao } 120\end{array}$ & $\begin{array}{l}\text { Hospital } \\
\text { information } \\
\text { system (HIS) }\end{array}$ & $\begin{array}{l}\text { Examining acceptance } \\
\text { of hospital information } \\
\text { systems (HIS) by } \\
\text { physicians }\end{array}$ & 2012 & $\begin{array}{l}\text { Physicians } \\
(N=81)\end{array}$ & $\begin{array}{l}\text { Hospital/System quality, } \\
\text { information quality, } \\
\text { service quality }\end{array}$ & Taiwan \\
\hline $\begin{array}{l}\text { Kim and } \\
\text { Park } 121\end{array}$ & $\begin{array}{l}\text { Health } \\
\text { information } \\
\text { technology } \\
(\mathrm{HIT})\end{array}$ & $\begin{array}{l}\text { Developing and verify } \\
\text { the extended } \\
\text { technology acceptance } \\
\text { model (TAM) in health } \\
\text { care }\end{array}$ & 2012 & $\begin{array}{l}\text { Health } \\
\text { consumers } \\
(n=728)\end{array}$ & $\begin{array}{l}\text { Home/Incorporating the } \\
\text { Health Belief Model } \\
\text { (HBM) and theory of } \\
\text { planned behavior (TPB), } \\
\text { along with the TAM }\end{array}$ & $\begin{array}{l}\text { South } \\
\text { Korea }\end{array}$ \\
\hline $\begin{array}{l}\text { Parra } \\
\text { et al }{ }^{122}\end{array}$ & $\begin{array}{l}\text { Care service for } \\
\text { the treatment } \\
\text { of acute stroke } \\
\text { patients based } \\
\text { on } \\
\text { telemedicine } \\
\text { (TeleStroke) }\end{array}$ & $\begin{array}{l}\text { Development, } \\
\text { implementation, and } \\
\text { evaluation of a care } \\
\text { service for the } \\
\text { treatment of acute } \\
\text { stroke patients based } \\
\text { on telemedicine } \\
\text { (TeleStroke) using a } \\
\text { TAM }\end{array}$ & 2012 & $\begin{array}{l}\text { Medical } \\
\text { profession- } \\
\text { als } \\
(N=34)\end{array}$ & $\begin{array}{l}\text { Hospital/Subjective } \\
\text { norm, facilitating } \\
\text { conditions }\end{array}$ & Spain \\
\hline $\begin{array}{l}\text { Gagnon } \\
\text { et al }\end{array}$ & $\begin{array}{l}\text { Telemonitoring } \\
\text { system }\end{array}$ & $\begin{array}{l}\text { Using a modified TAM } \\
\text { to evaluate health care } \\
\text { professionals' adoption } \\
\text { of a new } \\
\text { telemonitoring system }\end{array}$ & 2012 & $\begin{array}{l}\text { Health care } \\
\text { profession- } \\
\text { als } \\
(N=234)\end{array}$ & $\begin{array}{l}\text { Hospital/habit, } \\
\text { compatibility, } \\
\text { facilitators, subjective } \\
\text { norm }\end{array}$ & Spain \\
\hline Wangia $^{124}$ & $\begin{array}{l}\text { Immunization } \\
\text { registry }\end{array}$ & $\begin{array}{l}\text { Extending with } \\
\text { contextual factors } \\
\text { (contextualized TAM) } \\
\text { to test hypotheses } \\
\text { about immunization } \\
\text { registry usage }\end{array}$ & 2012 & $\begin{array}{l}\text { Immuniza- } \\
\text { tion } \\
\text { registry } \\
\text { end-users } \\
(n=100)\end{array}$ & $\begin{array}{l}\text { Unit of immunization } \\
\text { registry/job-task } \\
\text { change, commitment to } \\
\text { change, system interface } \\
\text { characteristic, subjective } \\
\text { norm, computer self- } \\
\text { efficacy }\end{array}$ & $\begin{array}{l}\text { United } \\
\text { States }\end{array}$ \\
\hline $\begin{array}{l}\text { Wong } \\
\text { et al }\end{array}$ & $\begin{array}{l}\text { Intelligent } \\
\text { Comprehen- } \\
\text { sive Interactive } \\
\text { Care (ICIC) } \\
\text { system } \\
\text { (Telemedical) }\end{array}$ & $\begin{array}{l}\text { Evaluating the users' } \\
\text { intention using a } \\
\text { modified technological } \\
\text { acceptance model } \\
\text { (TAM) }\end{array}$ & 2012 & $\begin{array}{l}\text { Elderly } \\
\text { people } \\
(N=121)\end{array}$ & $\begin{array}{l}\text { Elderly care/The TAM2 } \\
\text { theory and enjoyment } \\
\text { factor }\end{array}$ & Taiwan \\
\hline $\begin{array}{l}\text { Holden } \\
\text { et al }\end{array}$ & $\begin{array}{l}\text { Bar-coded } \\
\text { medication } \\
\text { administration } \\
(\mathrm{BCMA})\end{array}$ & $\begin{array}{l}\text { Identifying predictors } \\
\text { of nurses' acceptance } \\
\text { of bar-coded } \\
\text { medication } \\
\text { administration (BCMA) }\end{array}$ & 2012 & $\begin{array}{l}\text { Nurses } \\
(N=83)\end{array}$ & $\begin{array}{l}\text { Hospital/Social } \\
\text { influence, training, } \\
\text { technical support, age, } \\
\text { experience, satisfaction }\end{array}$ & $\begin{array}{l}\text { United } \\
\text { States }\end{array}$ \\
\hline $\begin{array}{l}\text { Dünnebeil } \\
\text { et al }\end{array}$ & $\begin{array}{l}\text { Electronic } \\
\text { health (e- } \\
\text { health) in } \\
\text { ambulatory } \\
\text { care } \\
\text { (Telemedicine) }\end{array}$ & $\begin{array}{l}\text { Extending technology } \\
\text { acceptance models } \\
\text { (TAMs) for electronic } \\
\text { health (e-health) in } \\
\text { ambulatory care } \\
\text { settings by physicians }\end{array}$ & 2012 & $\begin{array}{l}\text { Physicians } \\
(N=117)\end{array}$ & $\begin{array}{l}\text { Ambulatory care/ } \\
\text { building based on TAM } \\
\text { and UTAUT (process } \\
\text { orientation, importance } \\
\text { of standardization, e- } \\
\text { health knowledge, } \\
\text { importance of } \\
\text { documentation, } \\
\text { importance of data } \\
\text { security, intensity of IT } \\
\text { utilization) }\end{array}$ & Germany \\
\hline $\begin{array}{l}\text { Asua } \\
\text { et al }{ }^{128}\end{array}$ & $\begin{array}{l}\text { Telemonitor- } \\
\text { ing }\end{array}$ & $\begin{array}{l}\text { Examining the } \\
\text { psychosocial factors } \\
\text { related to } \\
\text { telemonitoring } \\
\text { acceptance among }\end{array}$ & 2012 & $\begin{array}{l}\text { Nurses, } \\
\text { general } \\
\text { practition- } \\
\text { ers, and }\end{array}$ & $\begin{array}{l}\text { Homecare/Habit, } \\
\text { compatibility, facilitator, } \\
\text { subjective norm }\end{array}$ & Spain \\
\hline
\end{tabular}




\begin{tabular}{|c|c|c|c|c|c|c|}
\hline Author(s) & $\begin{array}{l}\text { Technology } \\
\text { studied }\end{array}$ & Main topic & Years & Sample & $\begin{array}{l}\text { Setting/Incorporated } \\
\text { theories and variable } \\
\text { with the TAM }\end{array}$ & Country \\
\hline & & $\begin{array}{l}\text { health care based on } \\
\text { TAM2 }\end{array}$ & & $\begin{array}{l}\text { pediatri- } \\
\text { cians } \\
(N=268)\end{array}$ & & \\
\hline $\begin{array}{l}\text { Kummer } \\
\text { et } \mathrm{al}^{129}\end{array}$ & $\begin{array}{l}\text { Sensor-based } \\
\text { medication } \\
\text { administration } \\
\text { systems }\end{array}$ & $\begin{array}{l}\text { Usage of professional } \\
\text { ward nurses toward } \\
\text { sensor-based } \\
\text { medication systems } \\
\text { based on an TAM2 }\end{array}$ & 2013 & $\begin{array}{l}\text { Nurses } \\
(N=579)\end{array}$ & $\begin{array}{l}\text { Health associations/ } \\
\text { Qualitative overload, } \\
\text { quantitative overload, } \\
\text { personal innovativeness }\end{array}$ & Australia \\
\hline $\begin{array}{l}\text { Sedlmayr } \\
\text { et al }\end{array}$ & $\begin{array}{l}\text { Clinical } \\
\text { decision } \\
\text { support } \\
\text { systems for } \\
\text { medication }\end{array}$ & $\begin{array}{l}\text { Testing acceptance of } \\
\text { system by ED } \\
\text { physicians with TAM2 }\end{array}$ & 2013 & $\begin{array}{l}\text { Physicians } \\
(N=9)\end{array}$ & $\begin{array}{l}\text { Hospital/Resistance to } \\
\text { change(RTC), } \\
\text { compatibility (COM) }\end{array}$ & Germany \\
\hline $\begin{array}{l}\text { Abu- } \\
\text { Dalbouh }^{131}\end{array}$ & $\begin{array}{l}\text { Mobile health } \\
\text { applications }\end{array}$ & $\begin{array}{l}\text { Using TAM to evaluate } \\
\text { the system mobile } \\
\text { tracking model }\end{array}$ & 2013 & $\begin{array}{l}\text { Health care } \\
\text { profession- } \\
\text { als } \\
(N=-)\end{array}$ & $\begin{array}{l}\text {-/User satisfaction, } \\
\text { attribute of usability }\end{array}$ & $\begin{array}{l}\text { Saudi } \\
\text { Arabia }\end{array}$ \\
\hline $\begin{array}{l}\text { Tavakoli } \\
\text { et al }\end{array}$ & $\begin{array}{l}\text { Electronic } \\
\text { medical record } \\
(\text { EMR) }\end{array}$ & $\begin{array}{l}\text { Investigating the TAM } \\
\text { using EMR }\end{array}$ & 2013 & $\begin{array}{l}\text { Users of } \\
\text { EMR } \\
(n=\text { cen- } \\
\text { sus) }\end{array}$ & $\begin{array}{l}\text { Central Polyclinic Oil } \\
\text { Industry/data quality, } \\
\text { user interface }\end{array}$ & Iran \\
\hline $\begin{array}{l}\text { Buenes- } \\
\text { tado } \\
\text { et al }\end{array}$ & $\begin{array}{l}\text { Clinical } \\
\text { decision } \\
\text { support } \\
\text { systems } \\
\text { (CDSS) based } \\
\text { on } \\
\text { computerized } \\
\text { clinical } \\
\text { guidelines and } \\
\text { protocols } \\
\text { (CCGP) }\end{array}$ & $\begin{array}{l}\text { Determining } \\
\text { acceptance of initial } \\
\text { disposition of } \\
\text { physicians toward the } \\
\text { use of CDSS based on } \\
\text { (CCGP) }\end{array}$ & 2013 & $\begin{array}{l}\text { Physicians } \\
(N=8)\end{array}$ & $\begin{array}{l}\text { Hospital/ compatibility, } \\
\text { habits, facilitators, } \\
\text { subjective norm }\end{array}$ & Spain \\
\hline $\begin{array}{l}\text { Escobar- } \\
\text { Rodriguez } \\
\text { and } \\
\text { Bartual- } \\
\text { Sopena }\end{array}$ & $\begin{array}{l}\text { Enterprise } \\
\text { resources } \\
\text { planning (ERP) } \\
\text { systems }\end{array}$ & $\begin{array}{l}\text { Analyzing the attitude } \\
\text { of health care } \\
\text { personnel toward the } \\
\text { use of an ERP system in } \\
\text { public hospital }\end{array}$ & 2013 & $\begin{array}{l}\text { Health care } \\
\text { personnel } \\
(n=59)\end{array}$ & $\begin{array}{l}\text { Hospital/Experience } \\
\text { with IT, training, } \\
\text { support, age }\end{array}$ & Spain \\
\hline Su et al ${ }^{135}$ & $\begin{array}{l}\text { Telecare } \\
\text { systems }\end{array}$ & $\begin{array}{l}\text { Integrating patient } \\
\text { trust with the TAM to } \\
\text { explore the usage } \\
\text { intention model of } \\
\text { Telecare systems }\end{array}$ & 2013 & $\begin{array}{l}\text { Patients } \\
(N=365)\end{array}$ & $\begin{array}{l}\text { Hospital/Patient trust } \\
\text { (including Social Trust, } \\
\text { Institutional Trust) }\end{array}$ & Taiwan \\
\hline $\begin{array}{l}\text { Alali and } \\
\text { Juhana } 136\end{array}$ & $\begin{array}{l}\text { Virtual } \\
\text { communities of } \\
\text { practice (VCoPs) }\end{array}$ & $\begin{array}{l}\text { Exploring VCoPs } \\
\text { satisfaction based on } \\
\text { the technology } \\
\text { acceptance model } \\
\text { (TAM) and DeLone and } \\
\text { McLean IS success } \\
\text { model }\end{array}$ & 2013 & $\begin{array}{l}\text { Practition- } \\
\text { ers } \\
(N=112)\end{array}$ & $\begin{array}{l}\text { Hospital/Developing } \\
\text { from TAM and DeLone } \\
\text { and McLean IS success } \\
\text { models (knowledge } \\
\text { quality [KQ], system } \\
\text { quality [SyQ], service } \\
\text { quality [SeQ], } \\
\text { satisfaction [SAT]) }\end{array}$ & Malaysia \\
\hline $\begin{array}{l}\text { Wang } \\
\text { et al }\end{array}$ & Telecare system & $\begin{array}{l}\text { Using telecare system } \\
\text { to construct } \\
\text { medication safety } \\
\text { mechanisms for remote } \\
\text { area elderly uses TAM }\end{array}$ & 2013 & $\begin{array}{l}\text { Elderly } \\
\text { patients } \\
(N=271)\end{array}$ & $\begin{array}{l}\text { Remote areas/Person- } \\
\text { centered caring, } \\
\text { communication }\end{array}$ & Taiwan \\
\hline $\begin{array}{l}\text { Chen } \\
\text { et } \mathrm{al}^{138}\end{array}$ & & $\begin{array}{l}\text { Understanding the } \\
\text { influence on }\end{array}$ & 2013 & $\begin{array}{l}\text { Citizens } \\
(N=334)\end{array}$ & $\begin{array}{l}\text { Home/Relationship } \\
\text { quality (including trust, }\end{array}$ & Taiwan \\
\hline
\end{tabular}


Table 5 (Continued)

\begin{tabular}{|c|c|c|c|c|c|c|}
\hline Author(s) & $\begin{array}{l}\text { Technology } \\
\text { studied }\end{array}$ & Main topic & Years & Sample & $\begin{array}{l}\text { Setting/Incorporated } \\
\text { theories and variable } \\
\text { with the TAM }\end{array}$ & Country \\
\hline & $\begin{array}{l}\text { Hospital e- } \\
\text { appointment } \\
\text { system }\end{array}$ & $\begin{array}{l}\text { continuance intention } \\
\text { in the hospital } \\
\text { e-appointment system } \\
\text { based on extended } \\
\text { TAM }\end{array}$ & & & $\begin{array}{l}\text { satisfaction), } \\
\text { continuance intention }\end{array}$ & \\
\hline $\begin{array}{l}\text { Sicotte } \\
\text { et al }\end{array}$ & $\begin{array}{l}\text { Electronic } \\
\text { prescribing }\end{array}$ & $\begin{array}{l}\text { Identifying the factors } \\
\text { that can predict } \\
\text { physicians' use of } \\
\text { electronic prescribing } \\
\text { bases on expansion of } \\
\text { the technology } \\
\text { acceptance model } \\
\text { (TAM) }\end{array}$ & 2013 & $\begin{array}{l}\text { Physicians } \\
(N=61)\end{array}$ & $\begin{array}{l}\text { City region/Social } \\
\text { influence, practice } \\
\text { characteristics, } \\
\text { physician characteristics }\end{array}$ & Canada \\
\hline Liu et al $^{140}$ & $\begin{array}{l}\text { Web-based } \\
\text { personal } \\
\text { health record } \\
\text { system }\end{array}$ & $\begin{array}{l}\text { Extending TAM that } \\
\text { integrates the } \\
\text { physician-patient } \\
\text { relationship (PPR) } \\
\text { construct into TAM's } \\
\text { original constructs for } \\
\text { acceptance of Web- } \\
\text { based personal health } \\
\text { record system }\end{array}$ & 2013 & $\begin{array}{l}\text { Patients } \\
(N=50)\end{array}$ & $\begin{array}{l}\text { Medical center/ } \\
\text { Physician-patient } \\
\text { relationship (PPR) }\end{array}$ & Taiwan \\
\hline Ma et al ${ }^{141}$ & $\begin{array}{l}\text { Blended } \\
\text { e-learning } \\
\text { systems } \\
\text { (BELS) }\end{array}$ & $\begin{array}{l}\text { Integrating task- } \\
\text { technology fit (TTF), } \\
\text { computer self-efficacy, } \\
\text { the technology } \\
\text { acceptance model and } \\
\text { user satisfaction to } \\
\text { hypothesize a } \\
\text { theoretical model, to } \\
\text { explain and predict } \\
\text { user's behavioral } \\
\text { intention to use a BELS }\end{array}$ & 2013 & $\begin{array}{l}\text { Nurses } \\
(N=650)\end{array}$ & $\begin{array}{l}\text { Hospitals and medical } \\
\text { centers/Integrating the } \\
\text { TAM and task-technology } \\
\text { fit (TTF) }\end{array}$ & Taiwan \\
\hline $\begin{array}{l}\text { Escobar- } \\
\text { Rodríguez } \\
\text { and } \\
\text { Romero- } \\
\text { Alonso }^{142}\end{array}$ & $\begin{array}{l}\text { Automated } \\
\text { unit-based } \\
\text { medication } \\
\text { storage and } \\
\text { distribution } \\
\text { systems }\end{array}$ & $\begin{array}{l}\text { Identifying attitude of } \\
\text { nurses toward the use } \\
\text { of automated unit- } \\
\text { based medication } \\
\text { storage and } \\
\text { distribution systems } \\
\text { and influencing factors } \\
\text { bases on TAM }\end{array}$ & 2013 & $\begin{array}{l}\text { Nurses } \\
(N=118)\end{array}$ & $\begin{array}{l}\text { Hospital/Training, } \\
\text { perceived risk, experience } \\
\text { level }\end{array}$ & Spain \\
\hline Huang 143 & Telecare & $\begin{array}{l}\text { Exploring people's } \\
\text { intention to use } \\
\text { telecare with aid from } \\
\text { structural equation } \\
\text { modeling (SEM) } \\
\text { technique that is a } \\
\text { modification of TAM }\end{array}$ & 2013 & $\begin{array}{l}\text { People } \\
(N=369)\end{array}$ & $\begin{array}{l}\text { City region/ } \\
\text { Innovativeness, } \\
\text { subjective norm }\end{array}$ & Taiwan \\
\hline $\begin{array}{l}\text { Portela } \\
\text { et } \mathrm{al}^{144}\end{array}$ & $\begin{array}{l}\text { Pervasive } \\
\text { Intelligent } \\
\text { Decision } \\
\text { Support } \\
\text { System (PIDSS) }\end{array}$ & $\begin{array}{l}\text { Adopting of INTCare } \\
\text { system making use of } \\
\text { TAM3 in the ICU }\end{array}$ & 2013 & $\begin{array}{l}\text { Nurses } \\
(N=14)\end{array}$ & ICU/The TAM3 theory & Portugal \\
\hline $\begin{array}{l}\text { Johnson } \\
\text { et al }\end{array}$ & $\begin{array}{l}\text { Evidence- } \\
\text { adaptive } \\
\text { clinical } \\
\text { decision }\end{array}$ & $\begin{array}{l}\text { Acceptance of } \\
\text { evidence-adaptive } \\
\text { clinical decision } \\
\text { support system } \\
\text { associated with an }\end{array}$ & 2014 & $\begin{array}{l}\text { Internal } \\
\text { medicine } \\
\text { residents } \\
(N=44)\end{array}$ & $\begin{array}{l}\text { Hospital/User } \\
\text { satisfaction, computer } \\
\text { knowledge, general } \\
\text { optimism, self-reported } \\
\text { usage, usage trajectory }\end{array}$ & $\begin{array}{l}\text { United } \\
\text { States }\end{array}$ \\
\hline
\end{tabular}




\begin{tabular}{|c|c|c|c|c|c|c|}
\hline Author(s) & $\begin{array}{l}\text { Technology } \\
\text { studied }\end{array}$ & Main topic & Years & Sample & $\begin{array}{l}\text { Setting/Incorporated } \\
\text { theories and variable } \\
\text { with the TAM }\end{array}$ & Country \\
\hline & $\begin{array}{l}\text { support } \\
\text { system }\end{array}$ & $\begin{array}{l}\text { electronic health } \\
\text { record system using } \\
\text { TAM }\end{array}$ & & & $\begin{array}{l}\text { group, institutionalized } \\
\text { use }\end{array}$ & \\
\hline $\begin{array}{l}\text { Zhang } \\
\text { et al }\end{array}$ & Mobile health & $\begin{array}{l}\text { Assessment and } \\
\text { acceptance between } \\
\text { privacy and using } \\
\text { mobile health with aid } \\
\text { from TAM }\end{array}$ & 2014 & $\begin{array}{l}\text { Patients } \\
(N=489)\end{array}$ & $\begin{array}{l}\text { Hospital/ } \\
\text { Personalization, privacy }\end{array}$ & China \\
\hline $\begin{array}{l}\text { Andrews } \\
\text { et al }{ }^{147}\end{array}$ & $\begin{array}{l}\text { Personally } \\
\text { controlled } \\
\text { electronic } \\
\text { health record } \\
\text { (PCEHR) }\end{array}$ & $\begin{array}{l}\text { Examining how } \\
\text { individuals in the } \\
\text { general population } \\
\text { perceive the promoted } \\
\text { idea of having a PCEHR }\end{array}$ & 2014 & $\begin{array}{l}\text { Patients } \\
(N=750)\end{array}$ & $\begin{array}{l}\text { Homecare/Social norm, } \\
\text { privacy concern, trust, } \\
\text { perceived risk, } \\
\text { controllability, Web } \\
\text { self-efficacy, } \\
\text { compatibility, } \\
\text { perceived value }\end{array}$ & Australia \\
\hline $\begin{array}{l}\text { Gagnon } \\
\text { et al }\end{array}$ & $\begin{array}{l}\text { Electronic } \\
\text { health record } \\
(\mathrm{EHR})\end{array}$ & $\begin{array}{l}\text { Identifying the main } \\
\text { determinants of } \\
\text { physician acceptance of } \\
\text { EHR in a sample of } \\
\text { general practitioners } \\
\text { and specialists }\end{array}$ & 2014 & $\begin{array}{l}\text { Physicians } \\
(N=157)\end{array}$ & $\begin{array}{l}\text { Hospital/Integrating } \\
\text { original TAM, extended } \\
\text { TAM, psychosocial model }\end{array}$ & Canada \\
\hline $\begin{array}{l}\text { Hwang } \\
\text { et al }\end{array}$ & $\begin{array}{l}\text { Prehospital } \\
\text { telemetry }\end{array}$ & $\begin{array}{l}\text { Factors influencing the } \\
\text { acceptance of } \\
\text { telemetry by } \\
\text { emergency medical } \\
\text { technicians in } \\
\text { ambulances uses by } \\
\text { extended TAM }\end{array}$ & 2014 & $\begin{array}{l}\text { Emergency } \\
\text { medical } \\
\text { technicians } \\
(n=136)\end{array}$ & $\begin{array}{l}\text { Hospital/Job fit, loyalty, } \\
\text { organizational } \\
\text { facilitation, subjective } \\
\text { norm, expectation } \\
\text { confirmation, clinical } \\
\text { factors, nonclinical } \\
\text { factors }\end{array}$ & $\begin{array}{l}\text { South } \\
\text { Korea }\end{array}$ \\
\hline Tsai $^{150}$ & $\begin{array}{l}\text { Telehealth } \\
\text { system }\end{array}$ & $\begin{array}{l}\text { Integrating extended } \\
\text { TAM and health belief } \\
\text { model (HBM) for to } \\
\text { identify factors that } \\
\text { influence patients' } \\
\text { adoption to use } \\
\text { telehealth }\end{array}$ & 2014 & $\begin{array}{l}\text { Patients } \\
(N=365)\end{array}$ & $\begin{array}{l}\text { Home/Integrating } \\
\text { extended technology } \\
\text { acceptance model } \\
\text { (extended TAM) and } \\
\text { health belief model } \\
\text { (HBM) }\end{array}$ & Taiwan \\
\hline Rho et al ${ }^{151}$ & Telemedicine & $\begin{array}{l}\text { Developing } \\
\text { telemedicine service } \\
\text { acceptance model } \\
\text { based on the TAM with } \\
\text { the inclusion of three } \\
\text { predictive constructs } \\
\text { from the previously } \\
\text { published telemedicine } \\
\text { literature: (1) } \\
\text { accessibility of medical } \\
\text { records and of patients } \\
\text { as clinical factors, (2) } \\
\text { self-efficacy as an } \\
\text { individual factor, and } \\
\text { (3) perceived incentives } \\
\text { as regulatory factors }\end{array}$ & 2014 & $\begin{array}{l}\text { Physicians } \\
(N=183)\end{array}$ & $\begin{array}{l}\text { Medical centers and } \\
\text { hospitals/Self-efficacy, } \\
\text { accessibility, perceived } \\
\text { incentives }\end{array}$ & $\begin{array}{l}\text { South } \\
\text { Korea }\end{array}$ \\
\hline Tsai $^{152}$ & Telehealth & $\begin{array}{l}\text { Developing a } \\
\text { comprehensive } \\
\text { behavioral model for } \\
\text { analyzing the } \\
\text { relationships among } \\
\text { social capital factors } \\
\text { (social capital theory), } \\
\text { technological factors }\end{array}$ & 2014 & $\begin{array}{l}\text { End users } \\
\text { of a } \\
\text { telehealth } \\
\text { system } \\
(N=365)\end{array}$ & $\begin{array}{l}\text { City region/Integrating } \\
\text { social capital theory } \\
\text { (social trust, } \\
\text { institutional trust, social } \\
\text { participation), social } \\
\text { cognitive theory (system } \\
\text { self-efficacy) and TAM }\end{array}$ & Taiwan \\
\hline
\end{tabular}


Table 5 (Continued)

\begin{tabular}{|c|c|c|c|c|c|c|}
\hline Author(s) & $\begin{array}{l}\text { Technology } \\
\text { studied }\end{array}$ & Main topic & Years & Sample & $\begin{array}{l}\text { Setting/Incorporated } \\
\text { theories and variable } \\
\text { with the TAM }\end{array}$ & Country \\
\hline & & $\begin{array}{l}\text { (TAM), and system self- } \\
\text { efficacy (social } \\
\text { cognitive theory) in } \\
\text { telehealth }\end{array}$ & & & & \\
\hline $\begin{array}{l}\text { Horan } \\
\text { et al }{ }^{153}\end{array}$ & $\begin{array}{l}\text { Online } \\
\text { disability } \\
\text { evaluation } \\
\text { system }\end{array}$ & $\begin{array}{l}\text { Developing a } \\
\text { conceptual model for } \\
\text { physician acceptance } \\
\text { based on the TAM }\end{array}$ & 2004 & $\begin{array}{l}\text { Physicians } \\
(N=141)\end{array}$ & $\begin{array}{l}\text { Hospital/Organizational } \\
\text { readiness, technical } \\
\text { readiness, perceived } \\
\text { readiness, work practice } \\
\text { compatibility, social } \\
\text { demographics }\end{array}$ & $\begin{array}{l}\text { United } \\
\text { States }\end{array}$ \\
\hline $\begin{array}{l}\text { Saigí-Rubió } \\
\text { et al }\end{array}$ & Telemedicine & $\begin{array}{l}\text { Analyzing the } \\
\text { determinants of } \\
\text { telemedicine use in the } \\
\text { three countries with } \\
\text { TAM }\end{array}$ & 2014 & $\begin{array}{l}\text { Physicians } \\
(N=510)\end{array}$ & $\begin{array}{l}\text { Hospital, health care } \\
\text { centers of the urban } \\
\text { and rural/Optimism, } \\
\text { propensity to innovate, } \\
\text { level of ICT use }\end{array}$ & $\begin{array}{l}\text { Spain, } \\
\text { Colombia, } \\
\text { and Bolivia }\end{array}$ \\
\hline $\begin{array}{l}\text { Steininger } \\
\text { and } \\
\text { Barbara } 155\end{array}$ & $\begin{array}{l}\text { Electronic } \\
\text { health record } \\
(\text { EHR) }\end{array}$ & $\begin{array}{l}\text { Examining and } \\
\text { extending factors } \\
\text { influence acceptance } \\
\text { levels among } \\
\text { physicians, uses a } \\
\text { modified (TAM) }\end{array}$ & 2015 & $\begin{array}{l}\text { Physicians } \\
(N=204)\end{array}$ & $\begin{array}{l}\text { Hospital/Social impact, } \\
\text { HIT experience, privacy } \\
\text { concerns }\end{array}$ & Austria \\
\hline $\begin{array}{l}\text { Basak } \\
\text { et al }\end{array}$ & $\begin{array}{l}\text { Personal digital } \\
\text { assistant (PDA) }\end{array}$ & $\begin{array}{l}\text { Using an extended TAM } \\
\text { for exploring intention } \\
\text { to use personal digital } \\
\text { assistant (PDA) } \\
\text { technology among } \\
\text { physicians }\end{array}$ & 2015 & $\begin{array}{l}\text { Physicians } \\
(N=339)\end{array}$ & $\begin{array}{l}\text { Hospital/Integrating the } \\
\text { TAM and DeLone and } \\
\text { McLean IS success } \\
\text { models (knowledge } \\
\text { quality, system quality, } \\
\text { service quality and user } \\
\text { satisfaction) }\end{array}$ & Turkey \\
\hline $\begin{array}{l}\text { Al-Adwan } \\
\text { and } \\
\text { Hilary }^{157}\end{array}$ & $\begin{array}{l}\text { Electronic } \\
\text { health record } \\
(E H R)\end{array}$ & $\begin{array}{l}\text { Applying a modified } \\
\text { version of the revised } \\
\text { TAM to examine EHR } \\
\text { acceptance and } \\
\text { utilization by physicians }\end{array}$ & 2015 & $\begin{array}{l}\text { Physicians } \\
(N=227)\end{array}$ & $\begin{array}{l}\text { Hospital/Compatibility, } \\
\text { habit, subjective norm, } \\
\text { facilitators }\end{array}$ & Jordan \\
\hline $\begin{array}{l}\text { Kowitlawa- } \\
\text { kul et al }\end{array}$ & $\begin{array}{l}\text { Electronic } \\
\text { health record } \\
\text { for nursing } \\
\text { education } \\
\text { (EHRNE) }\end{array}$ & $\begin{array}{l}\text { Investigating the } \\
\text { factors influencing } \\
\text { nursing students' } \\
\text { acceptance of the EHRs } \\
\text { in nursing education } \\
\text { using the extended } \\
\text { TAM with self-efficacy } \\
\text { as a conceptual } \\
\text { framework }\end{array}$ & 2015 & $\begin{array}{l}\text { Students } \\
(N=212)\end{array}$ & Clinics/Self-efficacy & Singapore \\
\hline $\begin{array}{l}\text { Michel- } \\
\text { Verkerke } \\
\text { et al. }{ }^{59}\end{array}$ & $\begin{array}{l}\text { Patient record } \\
\text { development } \\
(\text { EPR) }\end{array}$ & $\begin{array}{l}\text { Developing a model } \\
\text { derived from the DOI } \\
\text { and TAM theory for } \\
\text { predicting EPR }\end{array}$ & 2015 & $\begin{array}{l}\text { Patients } \\
(N=-)\end{array}$ & $\begin{array}{l}\text {-/Derived from DOI and } \\
\text { TAM theory }\end{array}$ & $\begin{array}{l}\text { The } \\
\text { Nether- } \\
\text { lands }\end{array}$ \\
\hline $\operatorname{Lin}^{160}$ & $\begin{array}{l}\text { Hospital } \\
\text { information } \\
\text { system (HIS) }\end{array}$ & $\begin{array}{l}\text { Using the perspective } \\
\text { of TAM; national } \\
\text { cultural differences in } \\
\text { terms of masculinity/ } \\
\text { femininity, } \\
\text { individualism/ } \\
\text { collectivism, power } \\
\text { distance, and } \\
\text { uncertainty avoidance } \\
\text { are incorporated into } \\
\text { the TAM as moderators }\end{array}$ & 2015 & $\begin{array}{l}\text { Nurses } \\
(N=261)\end{array}$ & $\begin{array}{l}\text { Hospital/Power } \\
\text { distance, uncertainly } \\
\text { avoidance, masculinity } \\
\text { or femininity, } \\
\text { individualism or } \\
\text { collectivism, time } \\
\text { orientation }\end{array}$ & Taiwan \\
\hline
\end{tabular}




\begin{tabular}{|c|c|c|c|c|c|c|}
\hline Author(s) & $\begin{array}{l}\text { Technology } \\
\text { studied }\end{array}$ & Main topic & Years & Sample & $\begin{array}{l}\text { Setting/Incorporated } \\
\text { theories and variable } \\
\text { with the TAM }\end{array}$ & Country \\
\hline $\begin{array}{l}\text { Abdekhoda } \\
\text { et } \mathrm{al}^{59}\end{array}$ & $\begin{array}{l}\text { Electronic } \\
\text { medical } \\
\text { records (EMRs) }\end{array}$ & $\begin{array}{l}\text { Assessing physicians' } \\
\text { attitudes toward EMRs' } \\
\text { adoption by a } \\
\text { conceptual path model } \\
\text { of TAM and } \\
\text { organizational context } \\
\text { variables }\end{array}$ & 2015 & $\begin{array}{l}\text { Physicians } \\
(N=330)\end{array}$ & $\begin{array}{l}\text { Hospital/Management } \\
\text { support, training, } \\
\text { physicians' involvement, } \\
\text { physicians' autonomy, } \\
\text { doctor-patient } \\
\text { relationship }\end{array}$ & Iran \\
\hline $\begin{array}{l}\text { Gartrell } \\
\text { et al }\end{array}$ & $\begin{array}{l}\text { Electronic } \\
\text { personal } \\
\text { health records } \\
\text { (ePHRs) }\end{array}$ & $\begin{array}{l}\text { Using a modified } \\
\text { technology acceptance } \\
\text { model on nurses' } \\
\text { personal use of ePHRs }\end{array}$ & 2015 & $\begin{array}{l}\text { Nurses } \\
(N=847)\end{array}$ & $\begin{array}{l}\text { Hospital/Perceived data } \\
\text { privacy and security } \\
\text { protection, perceived } \\
\text { health-promoting role } \\
\text { model }\end{array}$ & $\begin{array}{l}\text { United } \\
\text { States }\end{array}$ \\
\hline $\begin{array}{l}\text { Carrera and } \\
\text { Lam- }^{162} \\
\text { booij }^{162}\end{array}$ & $\begin{array}{l}\text { Out-of-office } \\
\text { blood pressure } \\
\text { monitoring }\end{array}$ & $\begin{array}{l}\text { Developing an } \\
\text { analytical framework } \\
\text { based on the TAM, the } \\
\text { theory of planned } \\
\text { behavior, and the } \\
\text { model of personal } \\
\text { computing utilization } \\
\text { to guide the } \\
\text { implementation of out- } \\
\text { of-office BP monitoring } \\
\text { methods }\end{array}$ & 2015 & $\begin{array}{l}\text { Patients, } \\
\text { physicians } \\
(N=6)\end{array}$ & $\begin{array}{l}\text {-/Framework based on } \\
\text { the TAM, the TPB } \\
\text { (including self-efficiency, } \\
\text { social norm), and the } \\
\text { model of personal } \\
\text { computing utilization } \\
\text { (including enabling } \\
\text { conditions) }\end{array}$ & $\begin{array}{l}\text { The } \\
\text { Nether- } \\
\text { lands }\end{array}$ \\
\hline $\begin{array}{l}\text { Sieverdes } \\
\text { et al }\end{array}$ & $\begin{array}{l}\text { Mobile } \\
\text { technology }\end{array}$ & $\begin{array}{l}\text { Investigating kidney } \\
\text { transplant patients } \\
\text { attitudes and } \\
\text { perceptions toward } \\
\text { mobile technology with } \\
\text { aid from the } \\
\text { technology acceptance } \\
\text { model and self- } \\
\text { determination theory }\end{array}$ & 2015 & $\begin{array}{l}\text { Patients } \\
(N=57)\end{array}$ & $\begin{array}{l}\text { Medical center/ } \\
\text { Frameworks from the } \\
\text { TAM and self- } \\
\text { determination theory } \\
\text { (SDT) }\end{array}$ & $\begin{array}{l}\text { United } \\
\text { States }\end{array}$ \\
\hline $\begin{array}{l}\text { Song } \\
\text { et al }\end{array}$ & $\begin{array}{l}\text { Bar code } \\
\text { medication } \\
\text { administration } \\
\text { technology }\end{array}$ & $\begin{array}{l}\text { Using bar code } \\
\text { medication } \\
\text { administration } \\
\text { technology among } \\
\text { nurses in hospitals with } \\
\text { TAM }\end{array}$ & 2015 & $\begin{array}{l}\text { Nurses } \\
(N=163)\end{array}$ & $\begin{array}{l}\text { Hospital/Feedback and } \\
\text { communication about } \\
\text { errors, age, teamwork } \\
\text { within hospital units, } \\
\text { hospital management } \\
\text { support for patient } \\
\text { safety, nursing shift, } \\
\text { education, computer } \\
\text { skills, technology length } \\
\text { of use }\end{array}$ & $\begin{array}{l}\text { United } \\
\text { States }\end{array}$ \\
\hline $\begin{array}{l}\text { Jeon and } \\
\text { Park } 165\end{array}$ & $\begin{array}{l}\text { Mobile obesity- } \\
\text { management } \\
\text { applications } \\
\text { (apps) }\end{array}$ & $\begin{array}{l}\text { The acceptance of } \\
\text { mobile obesity- } \\
\text { management } \\
\text { applications (apps) by } \\
\text { the public were } \\
\text { analyzed using a mobile } \\
\text { health care system } \\
\text { (MHS) (TAM) }\end{array}$ & 2015 & $\begin{array}{l}\text { Public } \\
\text { (health } \\
\text { consumer) } \\
(N=94)\end{array}$ & $\begin{array}{l}\text { Homecare/ } \\
\text { Compatibility, self- } \\
\text { efficacy, technical } \\
\text { support and training }\end{array}$ & $\begin{array}{l}\text { South } \\
\text { Korea }\end{array}$ \\
\hline $\begin{array}{l}\text { Alrawab- } \\
\text { deh et al }^{166}\end{array}$ & $\begin{array}{l}\text { Electronic } \\
\text { health record } \\
(\mathrm{EHR})\end{array}$ & $\begin{array}{l}\text { The revealing factors } \\
\text { that affect the adoption } \\
\text { of EHR }\end{array}$ & 2015 & $\begin{array}{l}\text { Final users } \\
(N=6)\end{array}$ & $\begin{array}{l}\text { Health sector of NHS/ } \\
\text { Clinical safety, security, } \\
\text { integration, and } \\
\text { information sharing }\end{array}$ & $\begin{array}{l}\text { United Kin- } \\
\text { gdom }\end{array}$ \\
\hline $\begin{array}{l}\text { Escobar- } \\
\text { Rodríguez } \\
\text { and } \\
\text { Lourdes } 167\end{array}$ & $\begin{array}{l}\text { Enterprise } \\
\text { resources } \\
\text { planning (ERP) }\end{array}$ & $\begin{array}{l}\text { Impact of cultural } \\
\text { factors on user } \\
\text { attitudes toward ERP } \\
\text { use in public hospitals } \\
\text { and identifying }\end{array}$ & 2015 & $\begin{array}{l}\text { Users } \\
(N=59)\end{array}$ & $\begin{array}{l}\text { Hospital/Resistance to } \\
\text { be controlled, perceived } \\
\text { risks, resistance to } \\
\text { change }\end{array}$ & Spain \\
\hline
\end{tabular}


Table 5 (Continued)

\begin{tabular}{|c|c|c|c|c|c|c|}
\hline Author(s) & $\begin{array}{l}\text { Technology } \\
\text { studied }\end{array}$ & Main topic & Years & Sample & $\begin{array}{l}\text { Setting/Incorporated } \\
\text { theories and variable } \\
\text { with the TAM }\end{array}$ & Country \\
\hline & & $\begin{array}{l}\text { influencing factors uses } \\
\text { by TAM }\end{array}$ & & & & \\
\hline $\begin{array}{l}\text { Briz-Ponce } \\
\text { and García- } \\
\text { Peñalvo } 168\end{array}$ & $\begin{array}{l}\text { Mobile } \\
\text { technology } \\
\text { and "apps" }\end{array}$ & $\begin{array}{l}\text { Measurement and } \\
\text { explain the acceptance } \\
\text { of mobile technology } \\
\text { and "apps" in medical } \\
\text { education }\end{array}$ & 2015 & $\begin{array}{l}\text { Students, } \\
\text { medical } \\
\text { profession- } \\
\text { als } \\
(N=124)\end{array}$ & $\begin{array}{l}\text { University/Reliability, } \\
\text { social influence, } \\
\text { facilitating conditions, } \\
\text { self-efficacy, anxiety, } \\
\text { recommendation }\end{array}$ & Spain \\
\hline Lai et al ${ }^{169}$ & $\begin{array}{l}\text { Mobile hospital } \\
\text { registration } \\
\text { system }\end{array}$ & $\begin{array}{l}\text { The use of the mobile } \\
\text { hospital registration } \\
\text { system }\end{array}$ & 2015 & $\begin{array}{l}\text { Patients } \\
(N=501)\end{array}$ & $\begin{array}{l}\text { Hospital/Information } \\
\text { technology experience } \\
\text { (ITE) }\end{array}$ & Taiwan \\
\hline $\begin{array}{l}\text { Al-Nassar } \\
\text { et al }\end{array}$ & $\begin{array}{l}\text { Computerized } \\
\text { physician order } \\
\text { entry (CPOE) }\end{array}$ & $\begin{array}{l}\text { Behavior of CPOE } \\
\text { among physicians in } \\
\text { hospitals based on the } \\
\text { technology acceptance } \\
\text { model (TAM) }\end{array}$ & 2016 & $\begin{array}{l}\text { physicians } \\
(N=-)\end{array}$ & $\begin{array}{l}\text { Hospital/Instability of } \\
\text { new software providers, } \\
\text { software quality }\end{array}$ & Jordan \\
\hline Lin et $a^{171}$ & $\begin{array}{l}\text { Devices for } \\
\text { monitoring } \\
\text { elderly } \\
\text { people's } \\
\text { postures and } \\
\text { activities }\end{array}$ & $\begin{array}{l}\text { Designing and } \\
\text { development of a } \\
\text { novel, textile-based, } \\
\text { intelligent wearable } \\
\text { vest for real-time } \\
\text { posture monitoring and } \\
\text { emergency warnings }\end{array}$ & 2016 & $\begin{array}{l}\text { Elderly } \\
\text { people } \\
(N=50)\end{array}$ & $\begin{array}{l}\text { Homecare/Technology } \\
\text { anxiety }\end{array}$ & Taiwan \\
\hline $\begin{array}{l}\text { Suresh } \\
\text { et al }\end{array}$ & $\begin{array}{l}\text { Health } \\
\text { information } \\
\text { technology } \\
\text { (HIT) }\end{array}$ & $\begin{array}{l}\text { Analyzing the } \\
\text { application of the } \\
\text { technology acceptance } \\
\text { model (TAM) by } \\
\text { outpatients }\end{array}$ & 2016 & $\begin{array}{l}\text { Patients } \\
(N=200)\end{array}$ & $\begin{array}{l}\text { Hospital/Customized } \\
\text { information, } \\
\text { trustworthiness }\end{array}$ & India \\
\hline Ifinedo $^{173}$ & $\begin{array}{l}\text { Information } \\
\text { systems (ISs) }\end{array}$ & $\begin{array}{l}\text { The moderating effects } \\
\text { of demographic and } \\
\text { individual } \\
\text { characteristics on } \\
\text { nurses' acceptance of } \\
\text { information systems } \\
\text { (IS) }\end{array}$ & 2016 & $\begin{array}{l}\text { Nurses } \\
(N=197)\end{array}$ & $\begin{array}{l}\text { Hospital/Education, } \\
\text { computer knowledge }\end{array}$ & Canada \\
\hline $\begin{array}{l}\text { Goodarzi } \\
\text { et al }\end{array}$ & $\begin{array}{l}\text { Picture } \\
\text { archiving and } \\
\text { communica- } \\
\text { tion system } \\
\text { (PACS) }\end{array}$ & $\begin{array}{l}\text { The TAM has been used } \\
\text { to measure the } \\
\text { acceptance level of } \\
\text { PACS in the emergency } \\
\text { department }\end{array}$ & 2016 & $\begin{array}{l}\text { Users } \\
(N=\text { cen- } \\
\text { sus })\end{array}$ & Hospital/Change & Iran \\
\hline $\begin{array}{l}\text { Abdekhoda } \\
\text { et } \mathrm{al}^{175}\end{array}$ & $\begin{array}{l}\text { Electronic } \\
\text { medical } \\
\text { records (EMRs) }\end{array}$ & $\begin{array}{l}\text { Integrating a model to } \\
\text { explore physicians' } \\
\text { attitudes toward using } \\
\text { and accepting EMR in } \\
\text { health care }\end{array}$ & 2016 & $\begin{array}{l}\text { Physicians } \\
(N=330)\end{array}$ & $\begin{array}{l}\text { Hospital/Integrated TAM } \\
\text { and diffusion of } \\
\text { innovation theory (DOI) } \\
\text { model }\end{array}$ & Iran \\
\hline $\begin{array}{l}\text { Strudwick } \\
\text { et al }\end{array}$ & $\begin{array}{l}\text { Electronic } \\
\text { health record } \\
(\text { EHR) }\end{array}$ & $\begin{array}{l}\text { Developing integrated } \\
\text { TAM using theory of } \\
\text { reasoned action, theory } \\
\text { of planned behavior, } \\
\text { and the TAM to explain } \\
\text { behavior among nurses }\end{array}$ & 2016 & $\begin{array}{l}\text { Nurses } \\
(N=-)\end{array}$ & $\begin{array}{l}\text {-/Combining three } \\
\text { different models theory } \\
\text { of reasoned action } \\
\text { (TRA), theory of planned } \\
\text { behavior (TPB), and TAM }\end{array}$ & Canada \\
\hline $\begin{array}{l}\text { Hsiao and } \\
\text { Chen } 177\end{array}$ & $\begin{array}{l}\text { Computerized } \\
\text { clinical } \\
\text { practice } \\
\text { guidelines }\end{array}$ & $\begin{array}{l}\text { Investigating critical } \\
\text { factors influencing } \\
\text { physicians' intention } \\
\text { through an integrative } \\
\text { model of activity } \\
\text { theory, and the }\end{array}$ & 2016 & $\begin{array}{l}\text { Physicians } \\
(N=238)\end{array}$ & $\begin{array}{l}\text { Hospital/ incorporating } \\
\text { activity theory (three } \\
\text { dimensions of factors) } \\
\text { with TAM concepts } \\
\text { (intention as dependent } \\
\text { variable) }\end{array}$ & Taiwan \\
\hline
\end{tabular}




\begin{tabular}{|c|c|c|c|c|c|c|}
\hline Author(s) & $\begin{array}{l}\text { Technology } \\
\text { studied }\end{array}$ & Main topic & Years & Sample & $\begin{array}{l}\text { Setting/Incorporated } \\
\text { theories and variable } \\
\text { with the TAM }\end{array}$ & Country \\
\hline & & $\begin{array}{l}\text { technology acceptance } \\
\text { model }\end{array}$ & & & & \\
\hline $\begin{array}{l}\text { Saigi-Rubió } \\
\text { et al }{ }^{178}\end{array}$ & Telemedicine & $\begin{array}{l}\text { Investigating } \\
\text { determinants of } \\
\text { telemedicine use in } \\
\text { clinical practice among } \\
\text { medical professionals } \\
\text { using the TAM2 and } \\
\text { microdata }\end{array}$ & 2016 & $\begin{array}{l}\text { Physicians } \\
(N=96)\end{array}$ & $\begin{array}{l}\text { Health care institution/ } \\
\text { Security and } \\
\text { confidentiality, } \\
\text { subjective norm, } \\
\text { physician's relationship } \\
\text { with ICTs }\end{array}$ & Spain \\
\hline Lin et al $^{179}$ & $\begin{array}{l}\text { Nursing } \\
\text { information } \\
\text { system (NIS) }\end{array}$ & $\begin{array}{l}\text { Developing a } \\
\text { conceptual framework } \\
\text { that is based on the } \\
\text { technology acceptance } \\
\text { model } 3 \text { (TAM3) and } \\
\text { behavior theory }\end{array}$ & 2016 & $\begin{array}{l}\text { Nurses } \\
(N=245)\end{array}$ & $\begin{array}{l}\text { Hospital/Framework } \\
\text { that is based on the } \\
\text { TAM3 and behavior } \\
\text { theory (prior experience) }\end{array}$ & Taiwan \\
\hline $\begin{array}{l}\text { Ducey and } \\
\text { Coovert }^{180}\end{array}$ & $\begin{array}{l}\text { Tablet } \\
\text { computer }\end{array}$ & $\begin{array}{l}\text { Evaluating practicing } \\
\text { pediatricians to use of } \\
\text { tablet based on } \\
\text { extended technology } \\
\text { acceptance model }\end{array}$ & 2016 & $\begin{array}{l}\text { Pediatri- } \\
\text { cians } \\
\text { (physi- } \\
\text { cians) } \\
(N=261)\end{array}$ & $\begin{array}{l}\text { Hospital/Subjective } \\
\text { norm, compatibility, } \\
\text { reliability }\end{array}$ & $\begin{array}{l}\text { United } \\
\text { States }\end{array}$ \\
\hline $\begin{array}{l}\text { Holden } \\
\text { et al }\end{array}$ & $\begin{array}{l}\text { Novel health IT, } \\
\text { the large } \\
\text { customizable } \\
\text { interactive } \\
\text { monitor }\end{array}$ & $\begin{array}{l}\text { Examining pediatric } \\
\text { intensive care unit } \\
\text { nurses' perceptions, } \\
\text { acceptance, and use of } \\
\text { a novel health IT, the } \\
\text { large customizable } \\
\text { interactive monitor } \\
\text { bases on TAM2 }\end{array}$ & 2016 & $\begin{array}{l}\text { Nurses } \\
(N=167)\end{array}$ & $\begin{array}{l}\text { Hospital/Social } \\
\text { influence, perceived } \\
\text { training on system, } \\
\text { satisfaction with system, } \\
\text { complete use of system }\end{array}$ & $\begin{array}{l}\text { United } \\
\text { States }\end{array}$ \\
\hline $\begin{array}{l}\text { Omar } \\
\text { et al }\end{array}$ & $\begin{array}{l}\text { Prescribing } \\
\text { decision } \\
\text { support } \\
\text { systems } \\
\text { (EPDSS) }\end{array}$ & $\begin{array}{l}\text { Investigating } \\
\text { perception and use of } \\
\text { EPDSS at a tertiary care } \\
\text { using TAM2 }\end{array}$ & 2017 & $\begin{array}{l}\text { Physicians } \\
\text { (pediatri- } \\
\text { cians) } \\
(N=-)\end{array}$ & $\begin{array}{l}\text { Hospital/The TAM2 } \\
\text { theory }\end{array}$ & Sweden \\
\hline
\end{tabular}

Abbreviations: DOI, diffusion of innovation; HIV, human immunodeficiency virus; ICT, information and communication technology; ICU, intensive care unit; IS, information system; IT, information technology; NHS, National Health Service; USB, Universal Serial Bus; UTAUT, unified theory of acceptance and use of technology.

additions can be expected to allow comparisons between ICT application areas and harmonization between ICT applications and different organizational processes.

However, it has been suggested that a main reason for inconsistent predictive performance of the TAM in health services is the poor match between construct operationalization and the context in which the construct is measured, ${ }^{29}$ The second method to expand the TAM is to add contextualized TAM concepts that increase predictive power. One method to derive such contextualized concepts is belief elicitation ${ }^{60}$ which was also the process used to fit general behavioral theory to the ICT context when developing the TAM. ${ }^{20}$ However, this step-wise method is less suitable for comparisons between application areas and analyses of the organizational fit of new ICT applications from a general health service perspective. The results of this review suggest that consensus is needed upon how the TAM extension processes should be designed for uses in health services.
The primary threats to the validity of this review are concerned with the search strategy employed. First, it may be possible that we have not identified all relevant publications. The completeness of the search is dependent upon the search criteria used and the scope of the search, and is also influenced by the limitations of the search engines used. Publication bias is possibly a further threat to validity, in that we were primarily searching for literature available in the major computing digital libraries. It is possible that, as a result, we included more studies reporting positive results of the TAM as those publications reporting negative results are less likely to be published. Since we have been unable to undertake a formal metaanalysis, we are equally unable to undertake a funnel analysis-using a series of events that lead toward a defined goal-to investigate the possible extent of publication bias. Finally, it must be remembered that the TAM does not measure the benefit of ICT use, ${ }^{57}$ implying that measures of 
Table 6 The factors, variables, and theories used in common technological contexts in studies (respectively, repetition and importance)

\begin{tabular}{|c|c|c|c|c|}
\hline \multirow[t]{2}{*}{ Technology area } & \multicolumn{4}{|c|}{$\begin{array}{l}\text { Factors (variables) and intention-based theories incorporated to original TAM based on different user } \\
\text { groups and technological contexts }\end{array}$} \\
\hline & User groups & Factors and variables & $\begin{array}{l}\text { Intention-based } \\
\text { theories }\end{array}$ & $\begin{array}{l}\text { Extended TAM } \\
\text { version used }\end{array}$ \\
\hline \multirow[t]{3}{*}{$\begin{array}{l}\text { HIT systems in } \\
\text { general }\end{array}$} & $\begin{array}{l}\text { Health care } \\
\text { professionals }\end{array}$ & $\begin{array}{l}\text { Knowledge, endemic barriers, anxiety, } \\
\text { relevance, self-efficacy, subjective and } \\
\text { descriptive norms, age, image, job level, work } \\
\text { experience, computer skills, voluntariness, } \\
\text { information technology exposure, } \\
\text { innovativeness, online information } \\
\text { dependence }\end{array}$ & $\begin{array}{l}\text { DeLone and } \\
\text { McLean IS } \\
\text { success model }\end{array}$ & - \\
\hline & Nurses & $\begin{array}{l}\text { Social influence, perceived training on system, } \\
\text { satisfaction with system, complete use of } \\
\text { system }\end{array}$ & - & - \\
\hline & Patients & Customized information, trustworthiness. & $\begin{array}{l}\text { Health belief } \\
\text { model (HBM), } \\
\text { TPB }\end{array}$ & - \\
\hline \multirow{3}{*}{$\begin{array}{l}\text { Hospital } \\
\text { information } \\
\text { system (HIS) }\end{array}$} & Physicians & $\begin{array}{l}\text { System quality, information quality, service } \\
\text { quality }\end{array}$ & - & TAM3 \\
\hline & $\begin{array}{l}\text { Health care } \\
\text { professionals }\end{array}$ & $\begin{array}{l}\text { Compatibility, training, social influence, } \\
\text { facilitating condition, self-efficiency, anxiety }\end{array}$ & UTAUT & - \\
\hline & Nurses & $\begin{array}{l}\text { Power distance, uncertainly avoidance, } \\
\text { masculinity or femininity, individualism or } \\
\text { collectivism, time orientation, prior } \\
\text { experience, system quality, information } \\
\text { quality, self-efficacy, compatibility, top } \\
\text { management support, project team } \\
\text { competency }\end{array}$ & $\begin{array}{l}\text { Information } \\
\text { system success } \\
\text { model }\end{array}$ & TAM3 \\
\hline \multirow[t]{3}{*}{$\begin{array}{l}\text { Electronic health } \\
\text { record (EHR) }\end{array}$} & Physicians & $\begin{array}{l}\text { System acceptability, system characteristics, } \\
\text { organizational characteristics, individual } \\
\text { characteristics, system accessibility, } \\
\text { organizational cultural, perceptions of } \\
\text { institutional trust, perceived risk, information } \\
\text { integrity, social impact, HIT experience, } \\
\text { privacy concerns, compatibility, habit, } \\
\text { subjective norm, facilitators, management } \\
\text { support, training, physicians' involvement, } \\
\text { physicians' autonomy, doctor-patient } \\
\text { relationship }\end{array}$ & DOI, IDT, UTAUT & TAM2 \\
\hline & $\begin{array}{l}\text { Health care } \\
\text { professionals }\end{array}$ & $\begin{array}{l}\text { Perceived service level, perceived system } \\
\text { performance, data quality, user interface, self- } \\
\text { efficacy, clinical safety, security, integration } \\
\text { and information sharing } \\
\end{array}$ & - & - \\
\hline & Nurses & $\begin{array}{l}\text { Environment or context, nurse characteristics, } \\
\text { EHR characteristic }\end{array}$ & TRA, TPB, TTF & \\
\hline \multirow[t]{2}{*}{$\begin{array}{l}\text { e-Prescription } \\
\text { systems }\end{array}$} & Physicians & $\begin{array}{l}\text { Social influence, practice characteristics, } \\
\text { physician characteristics, perceived } \\
\text { compatibility, perceived usefulness to } \\
\text { enhance control systems, training, perceived } \\
\text { risks }\end{array}$ & - & - \\
\hline & Nurses & $\begin{array}{l}\text { Perceived compatibility, perceived usefulness } \\
\text { to enhance control systems, training, } \\
\text { perceived risks }\end{array}$ & - & - \\
\hline $\begin{array}{l}\text { Computers, } \\
\text { handheld (PDAs) }\end{array}$ & Physicians & $\begin{array}{l}\text { Subjective norm, compatibility, reliability, } \\
\text { knowledge quality, system quality, service } \\
\text { quality, user satisfaction, age, position in } \\
\text { hospital, cluster ownership, specialty }\end{array}$ & $\begin{array}{l}\text { DeLone and } \\
\text { McLean IS } \\
\text { success model }\end{array}$ & - \\
\hline
\end{tabular}




\begin{tabular}{|c|c|c|c|c|}
\hline \multirow[t]{5}{*}{ Technology area } & \multicolumn{4}{|c|}{$\begin{array}{l}\text { Factors (variables) and intention-based theories incorporated to original TAM based on different user } \\
\text { groups and technological contexts }\end{array}$} \\
\hline & User groups & Factors and variables & $\begin{array}{l}\text { Intention-based } \\
\text { theories }\end{array}$ & $\begin{array}{l}\text { Extended TAM } \\
\text { version used }\end{array}$ \\
\hline & $\begin{array}{l}\text { Health care } \\
\text { professionals }\end{array}$ & $\begin{array}{l}\text { Compatibility, support, personal } \\
\text { innovativeness, job relevance }\end{array}$ & - & - \\
\hline & Nurses & - & - & - \\
\hline & Pharmacists & $\begin{array}{l}\text { Subjective norm, image, output quality, result } \\
\text { demonstrability, job relevance, experience, } \\
\text { voluntariness }\end{array}$ & - & TAM2 \\
\hline \multirow[t]{4}{*}{ Telemedicine } & Physicians & $\begin{array}{l}\text { Security and confidentiality, relationship with } \\
\text { ICTs, subjective norm, facilitators, habit, } \\
\text { compatibility, self-efficacy, accessibility, } \\
\text { perceived incentives, process orientation, } \\
\text { importance of standardization, e-health } \\
\text { knowledge, importance of documentation, } \\
\text { importance of data, propensity to innovate, } \\
\text { organizational readiness, technical readiness, } \\
\text { social demographics, optimism, propensity to } \\
\text { innovate, enabling conditions }\end{array}$ & $\begin{array}{l}\text { UTAUT, TPB, } \\
\text { personal } \\
\text { computing } \\
\text { utilization }\end{array}$ & TAM2 \\
\hline & $\begin{array}{l}\text { Health care } \\
\text { professionals }\end{array}$ & $\begin{array}{l}\text { Subjective norm, job fit, loyalty, expectation } \\
\text { confirmation, clinical factors, nonclinical } \\
\text { factors, habit, compatibility, facilitators }\end{array}$ & - & - \\
\hline & Patients & $\begin{array}{l}\text { Patient trust, person-centered caring, } \\
\text { communication, enjoyment factor, social and } \\
\text { institutional trust, social participation, self- } \\
\text { efficacy, innovativeness, subjective norm, } \\
\text { social norm, enabling conditions, technology } \\
\text { anxiety }\end{array}$ & $\begin{array}{l}\text { HBM, social } \\
\text { capital theory, } \\
\text { social cognitive } \\
\text { theory, TPB, } \\
\text { personal } \\
\text { computing } \\
\text { utilization }\end{array}$ & TAM2 \\
\hline & Nurses & $\begin{array}{l}\text { Support from physicians, experience, support } \\
\text { from administrator. }\end{array}$ & - & - \\
\hline \multirow[t]{4}{*}{$\begin{array}{l}\text { Mobile } \\
\text { applications }\end{array}$} & Physicians & $\begin{array}{l}\text { Gender, experience, age, personal } \\
\text { innovativeness, compatibility, social influence }\end{array}$ & - & - \\
\hline & $\begin{array}{l}\text { Health care } \\
\text { professionals }\end{array}$ & $\begin{array}{l}\text { Reliability, social Influence, facilitating } \\
\text { conditions, self-efficacy, anxiety, } \\
\text { recommendation, user satisfaction, attribute } \\
\text { of usability, technical support and training, } \\
\text { compatibility }\end{array}$ & - & - \\
\hline & Nurses & $\begin{array}{l}\text { Subjective norm, image, output quality, result } \\
\text { demonstrability, job relevance, experience, } \\
\text { voluntariness }\end{array}$ & - & TAM2 \\
\hline & Patients & $\begin{array}{l}\text { Information technology experience (ITE), } \\
\text { compatibility, self-efficacy, technical support } \\
\text { and training, personalization, privacy, anxiety, } \\
\text { prior experience, person-centered, } \\
\text { communication }\end{array}$ & $\begin{array}{l}\text { Self- } \\
\text { determination } \\
\text { theory (SDT) }\end{array}$ & - \\
\hline $\begin{array}{l}\text { Personal health } \\
\text { record (PHR) }\end{array}$ & Patients & $\begin{array}{l}\text { Subjective norm, physician-patient } \\
\text { relationship (PPR), social norm, privacy } \\
\text { concern, trust, perceived risk, controllability, } \\
\text { self-efficacy, compatibility, perceived value }\end{array}$ & DOI & - \\
\hline
\end{tabular}

Abbreviations: DOI, diffusion of innovation; HIT, health information technology; ICT, information and communication technology; IDT, innovation diffusion theory; IS, information system; PDA, personal digital assistant; TAM, technology acceptance model; TPB, theory of planned behavior; TRA, theories of reasonable action; TTF, task-technology fit; UTAUT, unified theory of acceptance and use of technology. 
Table 7 Other models' comparison with TAM and confirmation of suitability of the TAM factors

\begin{tabular}{|c|c|c|c|c|c|c|}
\hline Author(s) & $\begin{array}{l}\text { Technology } \\
\text { studied }\end{array}$ & Main topic & Years & Sample & Setting & Country \\
\hline $\begin{array}{l}\text { Chau and } \\
\text { Jen-Hwa }\end{array}$ & Telemedicine & $\begin{array}{l}\text { Comparing different models, including } \\
\text { TAM, the theory of planned behavior } \\
\text { (TPB), and an integrated model for } \\
\text { acceptance telmedicine }\end{array}$ & 2002 & $\begin{array}{l}\text { Physicians } \\
(N>400)\end{array}$ & Hospital & China \\
\hline $\begin{array}{l}\text { Liang } \\
\text { et } \mathrm{al}^{51}\end{array}$ & $\begin{array}{l}\text { Computerized } \\
\text { physician order } \\
\text { entry (CPOE) }\end{array}$ & $\begin{array}{l}\text { Examining whether the TAM can be } \\
\text { applied to explain physician } \\
\text { acceptance of CPOE }\end{array}$ & 2006 & $\begin{array}{l}\text { Physicians } \\
(N=200)\end{array}$ & Hospital & China \\
\hline Day et $\mathrm{al}^{45}$ & $\begin{array}{l}\text { Videophone } \\
\text { technology }\end{array}$ & $\begin{array}{l}\text { Evaluating hospice providers } \ni \\
\text { attitudes and perceptions regarding } \\
\text { videophone technology in the hospice } \\
\text { setting in the context of the TAM }\end{array}$ & 2007 & $\begin{array}{l}\text { Providers } \\
(N=17)\end{array}$ & Hospice & Colombia \\
\hline $\begin{array}{l}\text { Smith and } \\
\text { Motley }^{50}\end{array}$ & $\begin{array}{l}\text { Electronic } \\
\text { prescribing }\end{array}$ & $\begin{array}{l}\text { The degree of e-prescribing } \\
\text { acceptance is highly predictable by } \\
\text { factors that are very stable ease-of-use } \\
\text { variables derived from the TAM }\end{array}$ & 2010 & $\begin{array}{l}\text { Pharma- } \\
\text { cists } \\
(N=50)\end{array}$ & $\begin{array}{l}\text { Pharma- } \\
\text { ceutical } \\
\text { company's } \\
\text { supply }\end{array}$ & $\begin{array}{l}\text { United } \\
\text { States }\end{array}$ \\
\hline Kim et $\mathrm{al}^{47}$ & $\begin{array}{l}\text { Telehomecare } \\
\text { (telemedicine) }\end{array}$ & $\begin{array}{l}\text { Comparing two theories of technology } \\
\text { adoption, the technology acceptance } \\
\text { model and the theory of planned } \\
\text { behavior, to explain and predict } \\
\text { physicians' acceptance and use of the } \\
\text { telehomecare technology }\end{array}$ & 2010 & $\begin{array}{l}\text { Physicians } \\
(N=40)\end{array}$ & Homecare & $\begin{array}{l}\text { United } \\
\text { States }\end{array}$ \\
\hline Kuo et al ${ }^{183}$ & $\begin{array}{l}\text { Mobile } \\
\text { electronic } \\
\text { medical record } \\
\text { (MEMR) } \\
\text { systems }\end{array}$ & $\begin{array}{l}\text { Confirming relationships between the } \\
\text { TAM components, and behavioral } \\
\text { intention in the technology acceptance } \\
\text { model toward MEMR usage }\end{array}$ & 2013 & $\begin{array}{l}\text { Nurses } \\
(N=665)\end{array}$ & Hospital & Taiwan \\
\hline $\begin{array}{l}\text { Manimaran } \\
\text { and } \\
\text { Lakshmi }^{49}\end{array}$ & $\begin{array}{l}\text { Health } \\
\text { management } \\
\text { information } \\
\text { system (HMIS) }\end{array}$ & $\begin{array}{l}\text { Formulating a model of technology } \\
\text { acceptance of health management } \\
\text { information system (HMIS) that } \\
\text { features the TAM was confirmed }\end{array}$ & 2013 & $\begin{array}{l}\text { Health } \\
\text { workers } \\
(N=960)\end{array}$ & $\begin{array}{l}\text { Rural } \\
\text { health care }\end{array}$ & India \\
\hline $\begin{array}{l}\text { Hsiao and } \\
\text { Tang } 44\end{array}$ & $\begin{array}{l}\text { Mobile health } \\
\text { care devices }\end{array}$ & $\begin{array}{l}\text { The use intention of mobile health care } \\
\text { devices from the perspectives of } \\
\text { elderly people }\end{array}$ & 2015 & $\begin{array}{l}\text { Elderly } \\
\text { people } \\
(N=338)\end{array}$ & - & Taiwan \\
\hline Kim et $\mathrm{al}^{48}$ & $\begin{array}{l}\text { Mobile } \\
\text { electronic } \\
\text { health records } \\
\text { (EMR) system }\end{array}$ & $\begin{array}{l}\text { Confirming the factors that influence } \\
\text { users' intentions to utilize a mobile } \\
\text { electronic health records (EMR) system } \\
\text { with TAM }\end{array}$ & 2016 & $\begin{array}{l}\text { Health care } \\
\text { profession- } \\
\text { als } \\
(N=942)\end{array}$ & Hospital & $\begin{array}{l}\text { South } \\
\text { Korea }\end{array}$ \\
\hline
\end{tabular}

Abbreviation: TAM, technology acceptance model.

technology acceptance and use intentions should not be mistaken for measures of technology value. Separate studies using measures of effectiveness or productivity are needed to assess the organizational value of the new technology.

The review was limited to those articles describing only the TAM and its application in health care service. By restricting our review to a narrow segment of this literature, we may have inadvertently eliminated meaningful details from other acceptance models and factors in health technologies acceptance. Also, there are books and book chapters that deal with the TAM in health care. These types of publications are not included in our review, but may contain information relevant to this review. Finally, our review includes only articles in English language and languages other than English might have information about the TAM in health care.

\section{Conclusion}

The result showed that telemedicine applications peaked between 1999 and 2017 and is the ICT application area most frequently studied using the TAM, implying that acceptance of telemedicine applications during this period was a major challenge when exploiting ICT to develop health service organizations. A majority of the reviewed articles reported extensions of the original TAM, suggesting that no optimal TAM version for use in health services has been established. Although the review results indicate a continuous progress, there are still areas that can be expanded and improved to increase the predictive performance of the TAM. Finally, it is suggested that the common investigated factors in the previous studies ( - Table 6 ), for each technological contexts and user groups, should tested empirically in real settings. If these factors confirmed, it is recommended that they will be 
applied as a basic model for each technological contexts and user groups.

\section{Clinical Relevance statement}

This systematic review showed that between 1999 and 2016, telemedicine applications were the ICT application area most frequently studied using the TAM, implying that acceptance of the telemedicine technology during this period was a major challenge for health service organizations. The construct validity of the model is showcased by its broad applicability to various technologies in health care. With the increasing number of technologies in the health care environment, the use of technology acceptance models is needed to guide implementation processes across health service contexts and user groups. This review has indicated continuous progress in revealing new aspects critical for ICT implementation having significant influence on health service processes and outcomes.

\section{Multiple Choice Questions}

1. Which of the following options are three main technological contexts using the TAM in health care ICTs?

a. (1) Hospital information system (HIS), (2) mobile applications, and (3) electronic health record (EHR).

b. (1) Telemedicine, (2) hospital information system (HIS), and (3) computers, handheld (PDAs).

c. (1) Telemedicine, (2) electronic health record (EHR), and (3) mobile applications.

d. (1) Electronic health record (EHR), (2) e-prescription systems, and (3) hospital information system (HIS).

Correct Answer: The correct answer is option c. The study identified three main technological contexts for using TAM in health care: (1) Telemedicine, (2) electronic health records (EHR), and (3) mobile applications. The geographical contexts of using TAM between different countries: Taiwan (telemedicine and mobile applications), U.S. and Iran (EHR), and Spain (telemedicine).

2. What variables can be added to the original TAM as a basis for model application in a variety of technological contexts? a. Subjective norm, self-efficacy, compatibility, experience, training, anxiety, habit, and facilitators.

b. Job relevance, age, communication, image, information quality, and uncertainty avoidance.

c. Power distance, time orientation, project team competency, acceptability, and organizational characteristics.

d. Training, management support, user interface, autonomy, cluster ownership, personal innovativeness, and loyalty.

Correct Answer: The correct answer is option a. The most common factors added to the original TAM in almost all technological contexts were, in order of importance and frequency of repetition, compatibility, subjective norm, selfefficacy, experience, training, anxiety, habit, and facilitators.
Protection of Human and Animal Subjects

Not applicable.

Funding

None.

\section{Conflict of Interest}

None.

\section{Acknowledgments}

This article was developed as a part of the research study code: 1395-01-52-2759 and by the supports of Urmia University of Medical Sciences. Also, we are very thankful to the editorial board of Applied Clinical Informatics journal for their valuable and constructive comments that made us very encouraged to reread and integrate all the comments.

\section{References}

1 Blackwell G, Gordon B. The future of IT in healthcare. Inform Health Soc Care 2008;33(04):211-326

2 Peña-López I. Improving health sector efficiency: the role of information and communication technologies. OECD Health Policy Studies; 2010

3 Scott RE. e-Records in health-preserving our future. Int J Med Inform 2007;76(5-6):427-431

4 Baker A. Crossing the quality chasm: a new health system for the 21st century. Br Med J 2001;323(7322):1192

5 Cooper JD. Organization, management, implementation and value of EHR implementation in a solo pediatric practice. J Healthc Inf Manag 2004;18(03):51-55

6 Rahimi B, Vimarlund V, Timpka T. Health information system implementation: a qualitative meta-analysis. J Med Syst 2009;33 (05):359-368

7 Hackl WO, Hoerbst A, Ammenwerth E. "Why the hell do we need electronic health records?". EHR acceptance among physicians in private practice in Austria: a qualitative study Methods Inf Med 2011;50(01):53-61

8 Nadri H, Rahimi B, Timpka T, Sedghi S. The top 100 articles in the medical informatics: a bibliometric analysis. J Med Syst 2017;41 (10): 150

9 Anderson JG. Clearing the way for physicians' use of clinical information systems. Commun ACM 1997;40(08):83-90

10 Jha AK, Ferris TG, Donelan K, et al. How common are electronic health records in the United States? A summary of the evidence. Health Aff (Millwood) 2006;25(06):w496-w507

11 Poon EG, Jha AK, Christino M, et al. Assessing the level of healthcare information technology adoption in the United States: a snapshot. BMC Med Inform Decis Mak 2006;6(01):1

12 Lorenzi NM, Novak LL, Weiss JB, Gadd CS, Unertl KM. Crossing the implementation chasm: a proposal for bold action. J Am Med Inform Assoc 2008;15(03):290-296

13 Rahimi B, Vimarlund V. Methods to evaluate health information systems in healthcare settings: a literature review. J Med Syst 2007;31(05):397-432

14 Catwell L, Sheikh A. Evaluating eHealth interventions: the need for continuous systemic evaluation. PLoS Med 2009;6(08): e1000126

15 Black AD, Car J, Pagliari C, et al. The impact of eHealth on the quality and safety of health care: a systematic overview. PLoS Med 2011;8(01):e1000387

16 Venkatesh V, Morris MG, Davis GB, Davis FD. User acceptance of information technology: Toward a unified view. Manage Inf Syst Q 2003;27:425-478 
17 Coiera E. Guide to Health Informatics. 3rd ed. London: CRC Press; 2015

18 Davis FD. Perceived usefulness, perceived ease of use, and user acceptance of information technology. Manage Inf Syst Q 1989; 13:319-340

19 Yousafzai SY, Foxall GR, Pallister JG. Technology acceptance: a meta-analysis of the TAM: part 1. J Model Manag 2007;2(03): 251-280

20 Yarbrough AK, Smith TB. Technology acceptance among physicians: a new take on TAM. Med Care Res Rev 2007;64(06): 650-672

21 Surendran P. Technology acceptance model: a survey of literature. Int J Business Soc Res 2013;2(04):4

22 Nadri H, Rahimi B, Lotfnezhad Afshar H, Samadbeik M, Garavand A. Factors affecting acceptance of hospital information systems based on extended technology acceptance model: a case study in three paraclinical departments. Appl Clin Inform 2018;9(02): 238-247

23 Holden RJ, Karsh BT. The technology acceptance model: its past and its future in health care. J Biomed Inform 2010;43(01):159-172

24 Hale JL, Householder BJ, Greene KL. The theory of reasoned action. In: Dillard JP, Pfau M, eds. The Persuasion Handbook: Developments in Theory and Practice. CA: SAGE Publications; 2002

25 Legris P, John I, Pierre C. Why do people use information technology? A critical review of the technology acceptance model. Inf Manage 2003;40(03):191-204

26 Davis FD, Bagozzi RP, Warshaw PR. User acceptance of computer technology: a comparison of two theoretical models. Manage Sci 1989;35(08):982-1003

27 Lee Y, Kozar KA, Rt LK. The technology acceptance model: past, present, and future. Comm Assoc Inform Syst 2003;12(01):50

28 Venkatesh V, Davis FD. A theoretical extension of the technology acceptance model: Four longitudinal field studies. Manage Sci 2000;46(02):186-204

29 Sharp JH. Development, extension, and application: a review of the technology acceptance model. Director 2006;5:7

30 King WR, Jun H. A meta-analysis of the technology acceptance model. Inf Manage 2006;43(06):740-755

31 Venkatesh V, Hillol B. Technology acceptance model 3 and a research agenda on interventions. Decis Sci 2008;39(02):273-315

32 Turner M, Barbara K, Pearl B, Stuart C, David B. Does the technology acceptance model predict actual use? A systematic literature review. Inf Softw Technol 2010;52(05):463-479

33 Hsiao $\mathrm{CH}$, Chyan Y. The intellectual development of the technology acceptance model: a co-citation analysis. Int J Inf Manage 2011;31(02):128-136

34 Hoyt RE, Yoshihashi A, Bailey NJ. Health informatics: practical guide for healthcare and information technology professionals. Informatics Education; 2014:533

35 Turban E, David K, Jae L, Dennis V. Electronic Commerce: A Managerial Perspective 2002. Prentice Hall; 2002. ISBN 013 (975285):4

36 Gagnon M-P, Desmartis M, Labrecque M, et al. Systematic review of factors influencing the adoption of information and communication technologies by healthcare professionals. J Med Syst 2012;36(01):241-277

37 Strudwick G. Predicting nurses' use of healthcare technology using the technology acceptance model: an integrative review. Comput Inform Nurs 2015;33(05):189-198, quiz E1

38 Ahlan AR, Isma'eel AB. An overview of patient acceptance of health information technology in developing countries: a review and conceptual model. Int J Inform Syst Project Management 2015;3(01):29-48

39 Garavand A, Mohseni M, Asadi H, Etemadi M, Moradi-Joo M, Moosavi A. Factors influencing the adoption of health information technologies: a systematic review. Electron Physician 2016; 8(08):2713-2718
40 Zanaboni P, Lettieri E. Institutionalizing telemedicine applications: the challenge of legitimizing decision-making. J Med Internet Res 2011;13(03):e72

41 Ventola CL. Mobile devices and apps for health care professionals: uses and benefits. P\&T 2014;39(05):356-364

$42 \mathrm{Hu}$ PJ, Chau Patrick YK, Sheng Olivia RL, Yan TK. Examining the technology acceptance model using physician acceptance of telemedicine technology. J Manage Inf Syst 1999;16(02): 91-112

43 Han S, Pekka M, Matti S, Markku K. Physicians' acceptance of mobile communication technology: an exploratory study. IJMC 2006;4(02):210-230

44 Hsiao C-H, Tang K-Y. Examining a model of mobile healthcare technology acceptance by the elderly in Taiwan. J Global Inform Tech Manag 2015;18(04):292-311

45 Day M, Demiris G, Oliver DP, Courtney K, Hensel B. Exploring underutilization of videophones in hospice settings. Telemed J E Health 2007;13(01):25-31

46 Chau PY, Jen-Hwa HP. Investigating healthcare professionals' decisions to accept telemedicine technology: an empirical test of competing theories. Inf Manage 2002;39(04):297-311

47 Kim J, Dellifraine JL, Dansky KH, Mccleary KJ. Physicians' acceptance of telemedicine technology: an empirical test of competing theories. Int J Inf Syst Change Manag 2010;4(03):210-225

$48 \mathrm{Kim} \mathrm{S}$, Lee $\mathrm{KH}$, Hwang $\mathrm{H}$, Yoo S. Analysis of the factors influencing healthcare professionals' adoption of mobile electronic medical record (EMR) using the unified theory of acceptance and use of technology (UTAUT) in a tertiary hospital. BMC Med Inform Decis Mak 2016;16(01):12

49 Manimaran S, Lakshmi KB. Development of model for assessing the acceptance level of users in rural healthcare system of Tamil Nadu, India. Technol Health Care 2013;21(05):479-492

50 Smith AD, Motley D. Operational and customer relationship management considerations of electronic prescribing among pharmacists. Int J Electron Healthc 2009;5(03):245-272

51 Liang $\mathrm{H}$, Xue Y, Wu X. User acceptance of computerized physician order entry: an empirical investigation. Int J Healthc Inf Syst Inform 2006;1(02):39-50(IJHISI)

52 Handayani PW, Hidayanto AN, Pinem AA, Hapsari IC, Sandhyaduhita PI, Budi I. Acceptance model of a Hospital Information System. Int J Med Inform 2017;99:11-28

53 May C, Harrison R, Finch T, MacFarlane A, Mair F, Wallace P; Telemedicine Adoption Study Group. Understanding the normalization of telemedicine services through qualitative evaluation. J Am Med Inform Assoc 2003;10(06):596-604

54 de Bont A, Bal R. Telemedicine in interdisciplinary work practices: on an IT system that met the criteria for success set out by its sponsors, yet failed to become part of every-day clinical routines. BMC Med Inform Decis Mak 2008;8(01):47

55 Wager KA, Lee FW, Glaser JP. Health Care Information Systems: A Practical Approach for Health Care Management. New York: John Wiley \& Sons; 2017

56 Chang $H$. Evaluation framework for telemedicine using the logical framework approach and a fishbone diagram. Healthc Inform Res 2015;21(04):230-238

57 Novak TP, Hoffman DL, Adam D. The influence of goal-directed and experiential activities on online flow experiences. J Consum Psychol 2003;13(01):3-16

58 Deci EL, Ryan RM. Self-determination theory. Handbook of Theories of Social Psychol 2011;1(2011):416-433

59 Abdekhoda M, Ahmadi M, Gohari M, Noruzi A. The effects of organizational contextual factors on physicians' attitude toward adoption of Electronic Medical Records. J Biomed Inform 2015; 53:174-179

60 Darker CD, French DP, Longdon S, Morris K, Eves FF. Are beliefs elicited biased by question order? A theory of planned behaviour belief elicitation study about walking in the UK general population. Br J Health Psychol 2007;12(Pt 1):93-110 
61 Barker DJ, van Schaik P, Simpson DS, Corbett WA. Evaluating a spoken dialogue system for recording clinical observations during an endoscopic examination. Med Inform Internet Med 2003; 28(02):85-97

62 Chang P, Hsu YS, Tzeng YM, Hou IC, Sang YY. Development and pilot evaluation of user acceptance of advanced mass-gathering emergency medical services PDA support systems. Stud Health Technol Inform 2004;107(Pt 2):1421-1425

63 Chang P, Hsu YS, Tzeng YM, Sang YY, Hou IC, Kao WF. The development of intelligent, triage-based, mass-gathering emergency medical service PDA support systems. J Nurs Res 2004;12 (03):227-236

64 Chen IJ, Yang KF, Tang FI, Huang CH, Yu S. Applying the technology acceptance model to explore public health nurses' intentions towards web-based learning: a cross-sectional questionnaire survey. Int J Nurs Stud 2008;45(06):869-878

65 Wilkins MA. Factors influencing acceptance of electronic health records in hospitals. Perspect Health Inf Manag 2009;6(05):1f

66 Marini SD, Hasman A, Huijer HA. Information technology for medication administration: assessing bedside readiness among nurses in Lebanon. Int J Evid-Based Healthc 2009;7(01):49-58

67 Van Schaik P, Bettany-Saltikov JA, Warren JG. Clinical acceptance of a low-cost portable system for postural assessment. Behav Inf Technol 2002;21(01):47-57

68 Huser V, Narus SP, Rocha RA. Evaluation of a flowchart-based EHR query system: a case study of RetroGuide. J Biomed Inform 2010;43(01):41-50

69 Cranen K, Veld RH, Ijzerman M, Vollenbroek-Hutten M. Change of patients' perceptions of telemedicine after brief use. Telemed J E Health 2011;17(07):530-535

70 Hung M-C, Jen WY. The adoption of mobile health management services: an empirical study. J Med Syst 2012;36(03):1381-1388

71 Aldosari B. User acceptance of a picture archiving and communication system (PACS) in a Saudi Arabian hospital radiology department. BMC Med Inform Decis Mak 2012;12(01):44

72 Noblin AM, Th WT, Myron F. Intention to use a personal health record: a theoretical analysis using the technology acceptance model. Int J Healthc Technol Manag 2013;14(1-2):73-89

73 Martínez-García A, Moreno-Conde A, Jódar-Sánchez F, Leal S, Parra C. Sharing clinical decisions for multimorbidity case management using social network and open-source tools. J Biomed Inform 2013;46(06):977-984

74 Monthuy-Blanc J, Bouchard S, Maïano C, Séguin M. Factors influencing mental health providers' intention to use telepsychotherapy in First Nations communities. Transcult Psychiatry 2013;50(02):323-343

75 Abdekhoda M, Ahmadi M, Dehnad A, Hosseini AF. Information technology acceptance in health information management. Methods Inf Med 2014;53(01):14-20

76 Cilliers L, Stephen F. User acceptance of telemedicine by health care workers: a case of the Eastern Cape province, South Africa. Electron J Inf Syst Dev Ctries 2014

77 Ologeanu-Taddei R, David M, Hugues D. Bourret R, eds. "Understanding the acceptance factors of an Hospital Information System: evidence from a French University Hospital." AMIA Annual Symposium Proceedings; American Medical Informatics Association; 2015

78 Money AG, Atwal A, Young KL, Day Y, Wilson L, Money KG. Using the Technology Acceptance Model to explore community dwelling older adults' perceptions of a 3D interior design application to facilitate pre-discharge home adaptations. BMC Med Inform Decis Mak 2015;15(01):73

79 Faruque MO, Holakouie Naieni K, Ardalan A, Ahmadnezhad E, Mohammadinia L. Feasibility assessment of using geoinformatics technology in disaster disease surveillance in a developing country, Iran. PLoS Curr 2015;7:7

80 Kivekäs E, Enlund H, Borycki E, Saranto K. General practitioners' attitudes towards electronic prescribing and the use of the national prescription centre. J Eval Clin Pract 2016;22(05): 816-825

81 Abdullah A, Liew SM, Hanafi NS, et al. What influences patients' acceptance of a blood pressure telemonitoring service in primary care? A qualitative study. Patient Prefer Adherence 2016; 10:99-106

82 Hanauer DA, Wu DTY, Yang L, et al. Development and empirical user-centered evaluation of semantically-based query recommendation for an electronic health record search engine. J Biomed Inform 2017;67:1-10

83 Rawstorne P, Rohan J, Peter C, eds. Issues in predicting and explaining usage behaviors with the technology acceptance model and the theory of planned behavior when usage is mandatory. Proceedings of the Twenty First International Conference on Information Systems. Association for Information Systems; 2000

84 Handy J, Hunter I, Whiddett R. User acceptance of inter-organizational electronic medical records. Health Informatics J 2001;7 (02):103-107

85 Chismar WG, Sonja W-P, eds. Test of the technology acceptance model for the internet in pediatrics. Proceedings of the AMIA Symposium. American Medical Informatics Association; 2002

86 Liang H, Xue Y, Anthony BT. PDA usage in healthcare professionals: testing an extended technology acceptance model. IJMC 2003;1(04):372-389

87 Liu L, Ma Q. The impact of service level on the acceptance of application service oriented medical records. Inf Manage 2005; 42(08):1121-1135

88 Liu L, Ma Q. Perceived system performance: a test of an extended technology acceptance model. ACM SIGMIS Database 2006;37 (2-3):51-59

89 Palm J-M, Isabelle C, Claude S, Patrice D. Determinants of user satisfaction with a clinical information system. AMIA Annual Symposium Proceedings; 2006:614-618. PubMed PMID: PMC1839744

90 Kim D, Chang H. Key functional characteristics in designing and operating health information websites for user satisfaction: an application of the extended technology acceptance model. Int J Med Inform 2007;76(11-12):790-800

$91 \mathrm{Wu}$ J-H, Wang S-C, Lin L-M. Mobile computing acceptance factors in the healthcare industry: a structural equation model. Int J Med Inform 2007;76(01):66-77

92 Tung F-C, Chang S-C, Chou C-M. An extension of trust and TAM model with IDT in the adoption of the electronic logistics information system in HIS in the medical industry. Int J Med Inform 2008;77(05):324-335

93 Lai T-Y, Larson EL, Rockoff ML, Bakken S. User acceptance of HIV TIDES-tailored interventions for management of depressive symptoms in persons living with HIV/AIDS. J Am Med Inform Assoc 2008;15(02):217-226

94 Wu J-H, Shen W-S, Lin L-M, Greenes RA, Bates DW. Testing the technology acceptance model for evaluating healthcare professionals' intention to use an adverse event reporting system. Int J Qual Health Care 2008;20(02):123-129

$95 \mathrm{Yu}$ P, Li H, Gagnon MP. Health IT acceptance factors in long-term care facilities: a cross-sectional survey. Int J Med Inform 2009;78 (04):219-229

96 Dasgupta A, Sansgiry SS, Sherer JT, Wallace D, Sikri S. Application of the Extended Technology Acceptance Model in predicting pharmacists' intention to use personal digital assistants. J Am Pharm Assoc (2003) 2009;49(06):792-796

97 Ilie V, Craig VS, Parikh MA, Courtney JF. Paper versus electronic medical records: the effects of access on physicians' decisions to use complex information technologies. Decis Sci 2009;40(02): 213-241

98 Trimmer K, Cellucci LW, Carla W, William W. Electronic medical records: TAM, UTAUT, and culture. Int J Healthc Inf Syst Inform 2009;4(03):55-68 
99 Lin S-P, Yang HY. Exploring key factors in the choice of e-health using an asthma care mobile service model. Telemed J E Health 2009;15(09):884-890

100 Aggelidis VP, Chatzoglou PD. Using a modified technology acceptance model in hospitals. Int J Med Inform 2009;78(02): $115-126$

101 Hyun S, Johnson SB, Stetson PD, Bakken S. Development and evaluation of nursing user interface screens using multiple methods. J Biomed Inform 2009;42(06):1004-1012

102 Vishwanath A, Brodsky L, Shaha S. Physician adoption of personal digital assistants (PDA): testing its determinants within a structural equation model. J Health Commun 2009;14(01):77-95

103 Morton ME, Susan W. EHR acceptance factors in ambulatory care: a survey of physician perceptions. EHR Acceptance Factors in Ambulatory Care: A Survey of Physician Perceptions/AHIMA. American Health Information Management Association; 2010

104 Zhang H, Cocosila M, Archer N. Factors of adoption of mobile information technology by homecare nurses: a technology acceptance model 2 approach. Comput Inform Nurs 2010;28 (01):49-56

105 Stocker G. Technology Acceptance of Electronic Medical Records by Nurses. Webster Groves, MO: Webster University; 2010

106 Lim S, Xue L, Yen CC, et al. A study on Singaporean women's acceptance of using mobile phones to seek health information. Int J Med Inform 2011;80(12):e189-e202

107 Schnall R, Bakken S. Testing the technology acceptance model: HIV case managers' intention to use a continuity of care record with context-specific links. Inform Health Soc Care 2011;36(03): 161-172

108 Kowitlawakul Y. The technology acceptance model: predicting nurses' intention to use telemedicine technology (eICU). Comput Inform Nurs 2011;29(07):411-418

109 Egea JMO, González MVR. Explaining physicians' acceptance of EHCR systems: an extension of TAM with trust and risk factors. Comput Human Behav 2011;27(01):319-332

110 Hsiao J-L, Chang H-C, Chen RF. A study of factors affecting acceptance of hospital information systems: a nursing perspective. J Nurs Res 2011;19(02):150-160

111 Orruño E, Gagnon MP, Asua J, Ben Abdeljelil A. Evaluation of teledermatology adoption by health-care professionals using a modified Technology Acceptance Model. J Telemed Telecare 2011;17(06):303-307

112 Melas CD, Zampetakis LA, Dimopoulou A, Moustakis V. Modeling the acceptance of clinical information systems among hospital medical staff: an extended TAM model. J Biomed Inform 2011;44 (04):553-564

113 Pai F-Y, Kai-I H. Applying the technology acceptance model to the introduction of healthcare information systems. Technol Forecast Soc Change 2011;78(04):650-660

114 Jimoh L, Pate MA, Lin L, Schulman KA. A model for the adoption of ICT by health workers in Africa. Int J Med Inform 2012;81(11): 773-781

115 Lu C-H, Hsiao J-L, Chen RF. Factors determining nurse acceptance of hospital information systems. Comput Inform Nurs 2012;30 (05):257-264

116 Lakshmi KB, Rajaram M. Impact of information technology reliance and innovativeness on rural healthcare services: study of Dindigul district in Tamil Nadu, India. Telemed J E Health 2012;18(05):360-370

117 Jian W-S, Syed-Abdul S, Sood SP, et al. Factors influencing consumer adoption of USB-based Personal Health Records in Taiwan. BMC Health Serv Res 2012;12(01):277

118 Escobar-Rodríguez T, Pedro M-L, Mercedes R-AM. Acceptance of e-prescriptions and automated medication-management systems in hospitals: an extension of the technology acceptance model. J Inf Syst 2012;26(01):77-96

119 Ketikidis P, Dimitrovski T, Lazuras L, Bath PA. Acceptance of health information technology in health professionals: an appli- cation of the revised technology acceptance model. Health Informatics J 2012;18(02):124-134

120 Chen R-F, Hsiao J-L. An empirical study of physicians' acceptance of hospital information systems in Taiwan. Telemed J E Health 2012;18(02):120-125

121 Kim J, Park HA. Development of a health information technology acceptance model using consumers' health behavior intention. J Med Internet Res 2012;14(05):e133

122 Parra C, Jódar-Sánchez F, Jiménez-Hernández MD, et al. Development, implementation, and evaluation of a telemedicine service for the treatment of acute stroke patients: teleStroke. Interact J Med Res 2012;1(02):e15

123 Gagnon MP, Orruño E, Asua J, Abdeljelil AB, Emparanza J. Using a modified technology acceptance model to evaluate healthcare professionals' adoption of a new telemonitoring system. Telemed J E Health 2012;18(01):54-59

124 Wangia $V$. Testing an extended theoretical framework to explain variance in use of a public health information system. Online J Public Health Inform 2012;4(03):ojphi.v4i3.4238

125 Wong AM, Chang W-H, Ke P-C, et al. Technology acceptance for an Intelligent Comprehensive Interactive Care (ICIC) system for care of the elderly: a survey-questionnaire study. PLoS One 2012; 7(08):e40591

126 Holden RJ, Brown RL, Scanlon MC, Karsh BT. Modeling nurses' acceptance of bar coded medication administration technology at a pediatric hospital. J Am Med Inform Assoc 2012;19(06): 1050-1058

127 Dünnebeil S, Sunyaev A, Blohm I, Leimeister JM, Krcmar H. Determinants of physicians' technology acceptance for e-health in ambulatory care. Int J Med Inform 2012;81(11):746-760

128 Asua J, Orruño E, Reviriego E, Gagnon MP. Healthcare professional acceptance of telemonitoring for chronic care patients in primary care. BMC Med Inform Decis Mak 2012;12(01):139

129 Kummer TF, Schäfer K, Todorova N. Acceptance of hospital nurses toward sensor-based medication systems: a questionnaire survey. Int J Nurs Stud 2013;50(04):508-517

130 Sedlmayr B, Patapovas A, Kirchner M, et al. Comparative evaluation of different medication safety measures for the emergency department: physicians' usage and acceptance of training, poster, checklist and computerized decision support. BMC Med Inform Decis Mak 2013;13(01):79

131 Abu-Dalbouh HM. A questionnaire approach based on the technology acceptance model for mobile tracking on patient progress applications. J Comput Sci 2013

132 Tavakoli N, Jahanbakhsh M, Shahin A, Mokhtari H, Rafiei M. Electronic medical record in Central Polyclinic of Isfahan oil industry: a case study based on technology acceptance model. Acta Inform Med 2013;21(01):23-25

133 Buenestado D, Elorz J, Pérez-Yarza EG, et al. Evaluating acceptance and user experience of a guideline-based clinical decision support system execution platform. J Med Syst 2013;37(02): 9910

134 Escobar-Rodriguez T, Bartual-Sopena L. The roles of users personal characteristics and organisational support in the attitude towards using ERP systems in a Spanish public hospital. Health Inf Manag 2013;42(01):18-28

135 Su S-P, Chung-Hung T, Hsu W-L. Extending the TAM model to explore the factors affecting intention to use telecare systems. JCP 2013;8(02):525-532

136 Alali H, Juhana S. Virtual communities of practice: the role of content quality and technical features to increase health care professionals' satisfaction. J Theoretical Appl Inform Tech 2013; 54(02):269-275

137 Wang J, Tsai C, Wang S. Using telecare system to construct medication safety mechanisms for remote area elderly. J Chem Pharm Res 2013;11(05):1-5

138 Chen S-C, Liu SC, Li SH, Yen DC. Understanding the mediating effects of relationship quality on technology acceptance: an 
empirical study of e-appointment system. J Med Syst 2013;37 (06):9981

139 Sicotte C, Taylor L, Tamblyn R. Predicting the use of electronic prescribing among early adopters in primary care. Can Fam Physician 2013;59(07):e312-e321

140 Liu C-F, Tsai YC, Jang FL. Patients' acceptance towards a webbased personal health record system: an empirical study in Taiwan. Int J Environ Res Public Health 2013;10(10):5191-5208

141 Ma C-M, Chao C-M, Bor-Wen C. Integrating technology acceptance model and task-technology fit into blended e-learning system. J Appl Sci (Faisalabad) 2013;13(05):736

142 Escobar-Rodríguez T, Romero-Alonso MM. Modeling nurses' attitude toward using automated unit-based medication storage and distribution systems: an extension of the technology acceptance model. Comput Inform Nurs 2013;31(05):235-243

143 Huang J-C. Innovative health care delivery system-a questionnaire survey to evaluate the influence of behavioral factors on individuals' acceptance of telecare. Comput Biol Med 2013;43 (04):281-286

144 Portela F, Filipe SM, Álvaro S, Fernando R, António A, José M. Adoption of pervasive intelligent information systems in intensive medicine. Proc Tech 2013;9:1022-1032

145 Johnson MP, Kai Z, Rema P. Modeling the longitudinality of user acceptance of technology with an evidence-adaptive clinical decision support system. Decis Support Syst 2014;57: 444-453

146 Zhang X, Guo X, Guo F, Lai KH. Nonlinearities in personalizationprivacy paradox in mHealth adoption: the mediating role of perceived usefulness and attitude. Technol Health Care 2014;22 (04):515-529

147 Andrews L, Gajanayake R, Sahama T. The Australian general public's perceptions of having a personally controlled electronic health record (PCEHR). Int J Med Inform 2014;83(12):889-900

148 Gagnon M-P, Ghandour K, Talla PK, et al. Electronic health record acceptance by physicians: testing an integrated theoretical model. J Biomed Inform 2014;48:17-27

149 Hwang JY, Kim KY, Lee KH. Factors that influence the acceptance of telemetry by emergency medical technicians in ambulances: an application of the extended technology acceptance model. Telemed J E Health 2014;20(12):1127-1134

150 Tsai C-H. The adoption of a Telehealth system: the integration of extended technology acceptance model and health belief model. J Med Imag Health Inform 2014;4(03):448-455

151 Rho MJ, Choi IY, Lee J. Predictive factors of telemedicine service acceptance and behavioral intention of physicians. Int J Med Inform 2014;83(08):559-571

152 Tsai C-H. Integrating social capital theory, social cognitive theory, and the technology acceptance model to explore a behavioral model of telehealth systems. Int J Environ Res Public Health 2014;11(05):4905-4925

153 Horan TA, Bengisu T, Brian H, Jacqueline B, eds. Use of online systems in clinical medical assessments: an analysis of physician acceptance of online disability evaluation systems. 2004 Proceedings of the 37th Annual Hawaii International Conference on System Sciences. IEEE; 2004

154 Saigí-Rubió F, Torrent-Sellens J, Jiménez-Zarco A. Drivers of telemedicine use: comparative evidence from samples of Spanish, Colombian and Bolivian physicians. Implement Sci 2014;9 (01):128

155 Steininger K, Barbara S. EHR acceptance among Austrian resident doctors. Health Policy Tech 2015;4(02):121-130

156 Basak E, Gumussoy CA, Calisir F. Examining the factors affecting PDA acceptance among physicians: an extended technology acceptance model. J Healthc Eng 2015;6(03):399-418

157 Al-Adwan AS, Hilary B. Exploring physicians' behavioural intention toward the adoption of electronic health records: an empirical study from Jordan. Int J Healthc Technol Manag 2015;15(02):89-111
158 Kowitlawakul Y, Chan SWC, Pulcini J, Wang W. Factors influencing nursing students' acceptance of electronic health records for nursing education (EHRNE) software program. Nurse Educ Today 2015;35(01):189-194

159 Michel-Verkerke MB, Stegwee RA, Spil Ton AM. The six P's of the next step in electronic patient records in the Netherlands. Health Policy Tech 2015;4(02):137-143

160 Lin H-C. The impact of national cultural differences on nurses' acceptance of hospital information systems. Comput Inform Nurs 2015;33(06):265-272

161 Gartrell K, Trinkoff AM, Storr CL, Wilson ML, Gurses AP. Testing the electronic personal health record acceptance model by nurses for managing their own health: a cross-sectional survey. Appl Clin Inform 2015;6(02):224-247

162 Carrera PM, Lambooij MS. Implementation of out-of-office blood pressure monitoring in the Netherlands: from clinical guidelines to patients' adoption of innovation. Medicine (Baltimore) 2015; 94(43):e1813

163 Sieverdes JC, Nemeth LS, Magwood GS, et al. Patient-centered mHealth living donor transplant education program for African Americans: development and analysis. JMIR Res Protoc 2015;4 (03):e84

164 Song L, Park B, Oh KM. Analysis of the technology acceptance model in examining hospital nurses' behavioral intentions toward the use of bar code medication administration. Comput Inform Nurs 2015;33(04):157-165

165 Jeon E, Park HA. Factors affecting acceptance of smartphone application for management of obesity. Healthc Inform Res 2015;21(02):74-82

166 Alrawabdeh W, Adel S, Fayiz S. Factors affecting the implementation of the national programme for information technology in the national health services: the case of Lorenzo in the North, Midlands and East of England region. Am J Appl Sci 2015;12(01):20

167 Escobar-Rodríguez T, Lourdes B-S. Impact of cultural factors on attitude toward using ERP systems in public hospitals. Rev Contabilidad 2015;18(02):127-137

168 Briz-Ponce L, García-Peñalvo FJ. An empirical assessment of a technology acceptance model for apps in medical education. J Med Syst 2015;39(11):176

169 Lai Y-H, Huang F-F, Yang HH. A study on the attitude of use the mobile clinic registration system in Taiwan. Technol Health Care 2015;24(Suppl 1):S205-S211

170 Al-Nassar BAY, Khalid Ali R, Sana'a Nawaf A-N. Impact of computerised physician order entry in Jordanian hospitals by using technology acceptance model. Int J Inf Syst Change Manag 2016;8(03):191-210

171 Lin W-Y, Chou W-C, Tsai TH, Lin CC, Lee MY. Development of a wearable instrumented vest for posture monitoring and system usability verification based on the technology acceptance model. Sensors (Basel) 2016;16(12):2172

172 Suresh V, Prabhakar K, Santhanalakshmi K, Maran K. Applying technology acceptance (TAM) model to determine the factors of acceptance in out-patient information system in private hospital sectors in Chennai city. J Pharm Sci Res 2016;8(12):1373-1377

173 Ifinedo P. The moderating effects of demographic and individual characteristics on nurses' acceptance of information systems: a Canadian study. Int J Med Inform 2016;87:27-35

174 Goodarzi H, Khatami SM, Javadzadeh H, et al. User acceptance of picture archiving and communication system in the emergency department. Iran J Radiol 2016;13(02):e20102

175 Abdekhoda M, Ahmadi M, Dehnad A, Noruzi A, Gohari M. Applying electronic medical records in health care physicians' perspective. Appl Clin Inform 2016;7(02):341-354

176 Strudwick G, Booth R, Mistry K. Can social cognitive theories help us understand nurses' use of electronic health records? Comput Inform Nurs 2016;34(04):169-174

177 Hsiao J-L, Chen RF. Critical factors influencing physicians' intention to use computerized clinical practice guidelines: an 
integrative model of activity theory and the technology acceptance model. BMC Med Inform Decis Mak 2016;16(01):3

178 Saigi-Rubió F, Jiménez-Zarco A, Torrent-Sellens J. Determinants of the intention to use telemedicine: evidence from primary care physicians. Int J Technol Assess Health Care 2016;32(1-2):29-36

179 Lin IC, Lin C, Hsu CL, Roan J, Yeh JS, Cheng YH. The usage behavior and intention stability of nurses: an empirical study of a nursing information system. J Nurs Res 2016;24(01):48-57

180 Ducey AJ, Coovert MD. Predicting tablet computer use: an extended Technology Acceptance Model for physicians. Health Policy Tech 2016;5(03):268-284
181 Holden RJ, Asan O, Wozniak EM, Flynn KE, Scanlon MC. Nurses' perceptions, acceptance, and use of a novel in-room pediatric ICU technology: testing an expanded technology acceptance model. BMC Med Inform Decis Mak 2016;16(01):145

182 Omar A, Johan E, Synnöve L, eds. Evaluation of Electronic Prescribing Decision Support System at a Tertiary Care Pediatric Hospital: The User Acceptance Perspective. ITCH; 2017

183 Kuo K-M, Liu CF, Ma CC. An investigation of the effect of nurses' technology readiness on the acceptance of mobile electronic medical record systems. BMC Med Inform Decis Mak 2013;13 (01):88 